\title{
Morphologies and ages of star cluster pairs and multiplets in the Small Magellanic Cloud ${ }^{\star}$
}

\author{
M.R. de Oliveira ${ }^{1}$, C.M. Dutra ${ }^{1}$, E. Bica ${ }^{1}$, and H. Dottori ${ }^{1}$ \\ Instituto de Fisica-UFRGS, CP. 15051, CEP 91501-970 POA - RS, Brazil
}

Received May 2; accepted June 21, 2000

\begin{abstract}
An isophotal atlas of 75 star cluster pairs and multiplets in the Small Magellanic Cloud is presented, comprising 176 objects. They are concentrated in the SMC main body. The isophotal contours were made from Digitized Sky Survey* images and showed relevant structural features possibly related to interactions in about $25 \%$ of the sample. Previous N-body simulations indicate that such shapes could be due to tidal tails, bridges or common envelopes. The diameter ratio between the members of a pair is preferentially in the range $1-2$, with a peak at 1 . The projected separation is in the range $\approx 3-22 \mathrm{pc}$ with a pronounced peak at $\approx 13$ pc. For 91 objects it was possible to derive ages from Colour-Magnitude Diagrams using the OGLE-II photometric survey. The cluster multiplets in general occur in OB stellar associations and/or HII region complexes. This indicates a common origin and suggests that multiplets coalesce into pairs or single clusters in a short time scale. Pairs in the SMC appear to be mostly coeval and consequently captures are a rare phenomenon. We find evidence that star cluster pairs and multiplets may have had an important role in the dynamical history of clusters presently seen as large single objects.
\end{abstract}

Key words: Magellanic Clouds — star clusters

Send offprint requests to: M.R. de Oliveira, e-mail: marcio@if.ufrgs.br

* The images in this study are based on photographic data obtained using the UK Schmidt Telescope, which was operated by the Royal Observatory Edinburgh, with funding from the UK Science and Engineering Research Council, until 1988 June, and thereafter by the Anglo-Australian Observatory. Original plate material is copyright by the Royal Observatory Edinburgh and the Anglo-Australian Observatory. The plates were processed into the present compressed digital form with their permission. The Digitized Sky Survey was produced at the Space Telescope Science Institute under US Government grant NAG W-2166.

\section{Introduction}

Star cluster pairs are common objects in the Magellanic Clouds and it is important to understand their formation and evolution processes. A list of 30 cluster pairs in the SMC was first presented by Hatzidimitriou \& Bhatia (1990). Bica \& Schmitt (1995, hereafter BS95) revised previous data on SMC extended objects (star clusters, associations and emission nebulae) and identified new ones using Sky Survey ESO/SERC $R$ and $J$ films. They presented a list of 40 pairs and 2 triple star clusters. Pietrzyński \& Udalski (1999a) reported 23 pairs and 4 triplets derived from Pietrzyński et al.'s (1998) star cluster catalogue in the OGLE survey area. Bica \& Dutra (2000) updated BS95's catalogue considering the entries in Pietrzyński et al.'s catalogue. Bica \& Dutra (2000) indicated 75 pairs and multiplets comprising 176 individual objects.

In recent years there has been growing evidence of interacting star clusters in the Magellanic Clouds, especially in the LMC. Bhatia \& Hatzidimitriou (1988) concluded that more than $50 \%$ of LMC pairs must be physical systems. Bhatia \& McGillivray (1988) found evidence that NGC 2214 is a merging binary star cluster, based on the presence of a flattened core and an extended halo. Indeed Sagar et al. (1991) detected two turnoff points revealing the presence of two interacting clusters. Bica et al. (1992) studied cluster pairs and multiplets in the LMC bar by means of integrated colours and found systems which resulted coeval and some with age differences. Vallenari et al. (1998) confirmed such scenarios by means of colourmagnitude diagrams. They also presented isophotal contours indicating physical interaction. Several other studies have found binarity evidence in LMC cluster pairs (e.g. Kontizas et al. 1993; Dieball \& Grebel 2000a, 2000b).

Bhatia et al. (1991) presented a photographic atlas of binary star cluster candidates in the LMC. For the SMC no morphological atlas is available and isophotal maps are required to test possible physical interactions. Indeed comparisons of isophotes with isopleth maps of N-body simulations proved to be a useful tool (Rodrigues et al. 1994; 
de Oliveira et al. 1998, hereafter ODB98), since the simulations produce features such as bridges, common envelopes and extensions. The observational importance of tidal tails as interaction signatures was also indicated by Leon et al. (1999).

In this work we provide isophotal maps for SMC pairs and multiplets to study the following properties of these candidate physical systems: (i) angular distribution; (ii) projected centre-to-centre separation of members; (iii) isophotal structures using the Digitized Sky Survey* (hereafter DSS); (iv) ages derived by means of isochrone fitting, when possible. We discuss candidate physical systems and infer a scenario for their formation and evolution.

In Sect. 2 we gather the objects providing coordinates, sizes, centre-to-centre separations and other details for the SMC pairs and multiplets. In Sect. 3 we provide the isophotal contour atlas together with classifications of interaction features whenever present. A preliminary version of the present isophotal SMC atlas together with one for the LMC was given in de Oliveira (1996). In Sect. 4 we derive cluster ages by means of Colour-Magnitude diagrams extracted from the OGLE-II $B V I$ photometric survey (Udalski et al. 1998). In Sect. 5 we discuss the properties of the systems and the possible scenarios for their origin and evolution. Finally, concluding remarks are given in Sect. 6.

\section{SMC pairs and multiplets}

Table 1 shows data for the 176 SMC objects which form 75 star cluster pairs and multiplets (Bica \& Dutra 2000), considering that the pair BS63/B67 in the latter study is possibly a triplet with NGC 294 (ODB98). By columns: (1) object cross-identification in the different catalogues: N (Henize 1956), K (Kron 1956), L (Lindsay 1958), H (Hodge 1960), SL (Shapley \& Lindsay 1963), B (Brück 1976), DEM (Davies et al. 1976), ESO (Lauberts 1982), H86- (Hodge 1986), MA (Meyssonier \& Azzopardi 1993), BS (Bica \& Schmitt 1995), OGLE (Pietrzyński et al. 1998). Note that some are embedded objects, named after the corresponding HII region; (2) and (3) right ascension and declination for the epoch J2000, respectively; (4) object type following BS95: C for star cluster, NC for small HII regions with embedded star clusters, CN for clusters which show some traces of emission, A for associations, $\mathrm{CA}$ and/or $\mathrm{AC}$ for star clusters of low density and objects with intermediate properties, AN for associations which show some traces of emission and NA for HII regions with embedded associations; (5) and (6) major and minor sizes, respectively; (7) position angle of major axis $\left(0^{\circ}=\mathrm{N}, 90^{\circ}=\mathrm{E}\right) ;(8)$ centre-to-centre angular separation $(1$ arcsec $=0.28$ pc assuming an absolute distance modulus $(m-M)=18.9$ for the SMC, Westerlund 1990). For triplets and multiplets we measured the separation between the two main members, as indicated in the corresponding object line in the table; (9) remarks: $m 6, m 5, m 4, m T$ and $m P$ indicate groups with six, five and four members, triplets and pairs, respectively. A running number identifies each candidate system. Abbreviations "br*" indicates that a bright star is present; "att" means attached to. A hierarchical indication is given for objects embedded in or superimposed on larger ones: "in" suggests a possible physical connection while "sup" suggests a projection. There are 56 pairs, 15 triplets, 2 quadruplets, 1 quintuplet and 1 sextuplet. The quintuplet is located in the star forming region NGC 395 with dimensions $25 \mathrm{pc} \times 18 \mathrm{pc}$ which in turn is embedded in the HII complex SMC-DEM126 with $66 \mathrm{pc} \times 35 \mathrm{pc}$ according to angular sizes in BS95. The sextuplet is mostly contained in the OB association H-A35 (Hodge 1985) with dimensions $57 \mathrm{pc} \times 47 \mathrm{pc}$. One quadruplet is located in H-A60 with $44 \mathrm{pc} \times 24 \mathrm{pc}$. Note that dimensions (Cols. 5 and 6) and position angle (Col. 7) are from Bica \& Dutra (2000) and were measured on plates. The dimensions are in general larger than the isophotal sizes in the subsequent analyses.

\section{The isophotal atlas}

For isophotal analysis purposes we extracted digitized images of pairs and multiplets from the DSS. The plates are from the SERC Southern Sky Survey and include IIIa-J long (3600 s), $V$ band medium (1200 s) and $V$ band short (300 s) exposures. The PDS pixel values correspond to photographic density measures from the original plates, and are not calibrated. The images were processed with the IRAF package at the Instituto de Física - UFRGS, applying a 2-d Gaussian filter to smooth out individual stars, producing isodensity maps.

We show the isophotal atlas of SMC star cluster pairs and multiplets in Figs. 1 throughout 7, where morphological evidence of interactions can be searched for. In each panel we point members and provide designations.

\subsection{Isophotal distortions}

Cluster pairs and multiplets with evidence of physical interaction are marked in Col. 6 of Table 2. Their isophotal maps show features such as isophotal distortions, common envelope, isophotal twistings, etc. We classified these isophotal maps according to the following criteria: (i) isophotes of the members are detached (d), but showing isophotal distortions; (ii) isophotes of the members are connected by a "bridge" (b); (iii) the members are embedded in the same isophotal envelope (e). These classification criteria are an important tool for a selection of interacting cluster pair candidates, since such isophotal morphologies are predominant in N-body model encounters (Rodrigues et al. 1994, ODB98). The classifications above were made for objects not disturbed by gas emission. It is worth noting that both bridges and envelopes 
Table 1. SMC pairs and multiplets

\begin{tabular}{|c|c|c|c|c|c|c|c|c|}
\hline Name & $\begin{array}{r}\mathrm{RA}(2000) \\
\mathrm{h}: \mathrm{m}: \mathrm{s}\end{array}$ & $\begin{array}{c}\operatorname{Dec}(2000) \\
\text { o(: : } " \text { " }\end{array}$ & $\mathrm{T}$ & $\begin{array}{r}\text { Dmax } \\
\prime \prime\end{array}$ & $\begin{array}{c}\text { Dmin } \\
\prime \prime\end{array}$ & $\begin{array}{c}\mathrm{PA} \\
\mathrm{O}\end{array}$ & $\begin{array}{r}\text { Separ. } \\
\prime \prime\end{array}$ & Comments \\
\hline (1) & (2) & (3) & (4) & (5) & (6) & (7) & (8) & (9) \\
\hline BS3 & $0: 30: 01$ & $-73: 20: 01$ & $\mathrm{AC}$ & 33 & 33 & - & 31 & $\mathrm{mT}-1$ \\
\hline H86-2 & $0: 30: 04$ & $-73: 20: 35$ & $\mathrm{AC}$ & 39 & 36 & 80 & 31 & $\mathrm{mT}-1$ \\
\hline BS4 & $0: 30: 07$ & $-73: 20: 58$ & C & 33 & 27 & $7^{70}$ & & $\mathrm{mT}-1$ \\
\hline BS7 & $0: 32: 43$ & $-73: 37: 59$ & $\mathrm{C}$ & 33 & 33 & $\therefore$ & 59 & $\mathrm{mP}-1$ \\
\hline BS8 & $0: 32: 56$ & $-73: 38: 33$ & $\mathrm{AC}$ & 36 & 36 & - & & $\mathrm{mP}-1$ \\
\hline H86-22 & $0: 34: 53$ & $-73: 02: 08$ & C & 18 & 18 & - & 42 & $\mathrm{mP}-2$ \\
\hline BS9 & $0: 35: 02$ & $-73: 02: 14$ & $\mathrm{AC}$ & 45 & 39 & 100 & & $\mathrm{mP}-2$ \\
\hline HW9 & $0: 36: 25$ & $-73: 00: 05$ & C & 45 & 45 & - & 52 & $\mathrm{mP}-3$ \\
\hline HW10 & $0: 36: 31$ & $-72: 59: 13$ & C & 57 & 57 & - & & $\mathrm{mP}-3$ \\
\hline B9 & $0: 37: 13$ & $-72: 57: 53$ & C & 27 & 27 & - & 68 & $\mathrm{mP}-4$ in $\mathrm{H}-\mathrm{A} 1$ \\
\hline H86-41 & $0: 37: 29$ & $-72: 57: 48$ & $\mathrm{C}$ & 33 & 24 & 50 & & $\mathrm{mP}-4$ \\
\hline H86-38 & $0: 37: 24$ & $-73: 01: 50$ & A & 72 & 72 & - & 46 & $\mathrm{mP}-5$ \\
\hline BS10 & $0: 37: 34$ & $-73: 01: 30$ & C & 39 & 39 & - & & $\mathrm{mP}-5$ \\
\hline BS14,SMC_OGLE165 & $0: 39: 12$ & $-73: 14: 46$ & C & 33 & 33 & - & 44 & $\mathrm{mP}-6$ \\
\hline SMC_OGLE5 & $0: 39: 22$ & $-73: 15: 28$ & $\mathrm{CA}$ & 51 & 45 & 90 & & $\mathrm{mP}-6$ \\
\hline $\mathrm{HW} 12 \mathrm{~A}$ & $0: 39: 26$ & $-73: 22: 59$ & C & 33 & 27 & 10 & 36 & $\mathrm{mP}-7$ \\
\hline H86-54 & $0: 39: 35$ & $-73: 22: 58$ & C & 33 & 33 & - & & $\mathrm{mP}-7$ \\
\hline B15 & $0: 39: 42$ & $-72: 58: 39$ & $\mathrm{CA}$ & 36 & 27 & 100 & 30 & $\mathrm{mP}-8$ \\
\hline H86-57 & $0: 39: 47$ & $-72: 58: 56$ & $\mathrm{CA}$ & 30 & 30 & - & & $\mathrm{mP}-8$ \\
\hline BS13 & $0: 40: 08$ & $-72: 45: 30$ & $\mathrm{CA}$ & 54 & 45 & 40 & 48 & $\mathrm{mP}-9$ \\
\hline BS248 & $0: 40: 15$ & $-72: 46: 02$ & $\mathrm{AC}$ & 39 & 30 & 70 & & $\mathrm{mP}-9$ \\
\hline $\mathrm{NGC} 220, \mathrm{~K} 18, \mathrm{~L} 22, \mathrm{ESO} 29 \mathrm{SC} 3$ & $0: 40: 31$ & $-73: 24: 10$ & $\mathrm{C}$ & 72 & 72 & - & 88 & $\mathrm{mT}-2$, in H-A3 \& SMC_OGLE8 \\
\hline NGC 222,K19,L24,ESO $29 \mathrm{SC} 4$ & $0: 40: 44$ & $-73: 23: 00$ & C & 72 & 72 & - & 88 & $\mathrm{mT}-2$, in H-A3 \& SMC_OGLE9 \\
\hline B23,SMC_OGLE170 & $0: 40: 55$ & $-73: 24: 07$ & C & 36 & 30 & 40 & & $\mathrm{mT}-2$, in $\mathrm{H}-\mathrm{A} 3$ \\
\hline B19 & $0: 40: 44$ & $-73: 03: 42$ & C & 27 & 21 & 170 & 32 & $\mathrm{mT}-3$ \\
\hline В20 & $0: 40: 49$ & $-73: 04: 09$ & C & 18 & 18 & - & 32 & $\mathrm{mT}-3$ \\
\hline H86-62,SMC_OGLE10 & $0: 40: 48$ & $-73: 05: 17$ & $\mathrm{C}$ & 36 & 36 & - & & $\mathrm{mT}-3$ \\
\hline $\mathrm{NGC} 231, \mathrm{~K} 20, \mathrm{~L} 25, \mathrm{ESO} 29 \mathrm{SC} 5$ & $0: 41: 06$ & $-73: 21: 07$ & C & 108 & 108 & - & 36 & mP-10, in H-A3 \& SMC_OGLE11 \\
\hline BS15 & $0: 41: 21$ & $-73: 20: 31$ & A & 138 & 138 & - & & $\mathrm{mP}-10$, in $\mathrm{H}-\mathrm{A} 3$ \\
\hline B21,SMC_OGLE171 & $0: 41: 15$ & $-72: 49: 55$ & c & 21 & 21 & - & 34 & mP-11 \\
\hline B22 & $0: 41: 21$ & $-72: 49: 32$ & $\mathrm{c}$ & 21 & 21 & - & & $\mathrm{mP}-11$ \\
\hline В29 & $0: 42: 11$ & $-73: 43: 51$ & $\mathrm{CA}$ & 30 & 21 & 100 & 46 & $\mathrm{mP}-12$ \\
\hline HW16,SMC_OGLE13 & $0: 42: 22$ & $-73: 44: 03$ & $\mathrm{CN}$ & 36 & 36 & - & & $\mathrm{mP}-12$, in SMC-DEM 7 \\
\hline $\mathrm{NGC} 241, \mathrm{~K} 22 \mathrm{w}, \mathrm{L} 29 \mathrm{~W}$ & $0: 43: 33$ & $-73: 26: 25$ & $\mathrm{C}$ & 57 & 57 & - & 23 & mP-13 \& ESO 29SC6w,SMC_OGLE17 \\
\hline $\mathrm{NGC} 242, \mathrm{~K} 22 \mathrm{e}, \mathrm{L} 29 \mathrm{e}$ & $0: 43: 38$ & $-73: 26: 37$ & C & 45 & 45 & - & & mP-13 \& ESO 29SC6e,BH1,SMC_OGLE18 \\
\hline B31,SMC_OGLE19,SMC_OGLE175 & $0: 43: 38$ & $-72: 57: 31$ & C & 30 & 24 & 150 & & $\mathrm{mT}-4$ \\
\hline BS20,SMC_OGLE20 & $0: 43: 38$ & $-72: 58: 48$ & C & 27 & 27 & - & 30 & $\mathrm{mT}-4$ \\
\hline H86-70,SMC_OGLE21 & $0: 43: 44$ & $-72: 58: 36$ & $\mathrm{C}$ & 39 & 27 & 50 & 30 & $\mathrm{mT}-4$ \\
\hline BS27,SMC_OGLE177 & $0: 44: 55$ & $-73: 10: 27$ & $\mathrm{C}$ & 24 & 21 & 80 & 19 & $\mathrm{mP}-14$, in $\mathrm{H} 86-72$ \\
\hline SMC-N10,L61-60,SMC-DEM11, & $0: 44: 56$ & $-73: 10: 11$ & $\mathrm{NC}$ & 21 & 21 & - & & $\mathrm{mP}-14$, in $\mathrm{H} 86-72 \&$ MA 85 \\
\hline NGC 248n,SMC-N13B,L61-67n & $0: 45: 24$ & $-73: 22: 34$ & NA & 42 & 36 & 110 & 21 & mP-15 \& SMC-DEM16n,ESO 29EN8n,MA101,SMC_OGLE26n \\
\hline NGC 248s,SMC-N13A,L61-67s & $0: 45: 26$ & $-73: 23: 04$ & $\mathrm{NC}$ & 42 & 33 & 150 & & mP-15 \& SMC-DEM16s,ESO 29EN8s,MA103,SMC_OGLE26s \\
\hline B39,SMC_OGLE27 & $0: 45: 26$ & $-73: 28: 53$ & C & 33 & 33 & - & 15 & mP-16 \\
\hline BS30 & $0: 45: 30$ & $-73: 29: 06$ & $\mathrm{C}$ & 24 & 24 & - & & $\mathrm{mP}-16$ \\
\hline в36 & $0: 45: 44$ & $-72: 50: 35$ & $\mathrm{C}$ & 42 & 36 & 140 & 10 & $\mathrm{mP}-17$ \\
\hline SMC_OGLE31 & $0: 45: 51$ & $-72: 50: 25$ & $\mathrm{CA}$ & 33 & 27 & 90 & & $\mathrm{mP}-17$, not B 36 \\
\hline В38 & $0: 45: 54$ & $-72: 36: 08$ & $\mathrm{C}$ & 18 & 18 & - & 38 & $\mathrm{mP}-18$ \\
\hline н86-79 & $0: 45: 58$ & $-72: 35: 36$ & C & 27 & 27 & - & & $\mathrm{mP}-18$ \\
\hline H86-76,SMC_OGLE182 & $0: 46: 02$ & $-73: 23: 44$ & C & 27 & 27 & - & & $\mathrm{mT}-5$,in SMC-DEM21 \\
\hline H86-78n,SMC_OGLE33n & $0: 46: 12$ & $-73: 23: 27$ & $\mathrm{CN}$ & 27 & 27 & - & 16 & mT-5, in SMC-N16 \\
\hline H86-78s,SMC_OGLE33s & $0: 46: 12$ & $-73: 23: 39$ & $\mathrm{CN}$ & 27 & 24 & 60 & 16 & mT-5, in SMC-N16 \\
\hline L31,SMC_OGLE36 & $0: 46: 35$ & $-72: 44: 32$ & $\mathrm{C}$ & 66 & 51 & - & & $\mathrm{mT}-6$ \\
\hline H86-83,SMC_OGLE35 & $0: 46: 34$ & $-72: 46: 26$ & $\mathrm{C}$ & 42 & 42 & - & 30 & $\mathrm{mT}-6$ \\
\hline H86-84,SMC_OGLE185 & $0: 46: 34$ & $-72: 45: 56$ & c & 24 & 24 & - & 30 & $\mathrm{mT}-6$ \\
\hline H86-86,SMC_OGLE40 & $0: 47: 01$ & $-73: 23: 35$ & C & 48 & 39 & 110 & 71 & $\mathrm{mP}-19$, in $\mathrm{H}-\mathrm{A} 9$ \\
\hline H86-87,SMC_OGLE187 & $0: 47: 06$ & $-73: 22: 17$ & C & 48 & 42 & 90 & & $\mathrm{mP}-19$, in $\mathrm{H}-\mathrm{A} 9$ \\
\hline H86-95 & $0: 47: 37$ & $-73: 00: 51$ & $\mathrm{CA}$ & 21 & 12 & 100 & 30 & $\mathrm{mP}-20$ \\
\hline н86-96 & $0: 47: 44$ & $-73: 00: 46$ & C & 18 & 18 & - & & $\mathrm{mP}-20$ \\
\hline BS35,SMC_OGLE42 & $0: 47: 50$ & $-73: 28: 42$ & C & 42 & 42 & - & 55 & $\mathrm{mP}-21$ \\
\hline K25,L35,SMC_OGLE45 & $0: 48: 01$ & $-73: 29: 10$ & C & 72 & 72 & - & & $\mathrm{mP}-21$ \\
\hline MA205 & $0: 48: 07$ & $-73: 14: 49$ & $\mathrm{NC}$ & 15 & 15 & - & 37 & $\mathrm{mT}-7$ \\
\hline SMC-N25,L61-106,SMC-DEM38, & $0: 48: 09$ & $-73: 14: 19$ & $\mathrm{NA}$ & 51 & 51 & - & & mT-7 \& MA208,SMC_OGLE189 \\
\hline SMC-N26,L61-107,MA 206 & $0: 48: 08$ & $-73: 14: 53$ & $\mathrm{NC}$ & 27 & 27 & - & 37 & $\mathrm{mT}-7$ \\
\hline H86-99,SMC_OGLE190 & $0: 48: 13$ & $-72: 47: 35$ & $\mathrm{CA}$ & 39 & 39 & - & 27 & $\mathrm{mP}-22$ \\
\hline H86-100,SMC_OGLE191 & $0: 48: 20$ & $-72: 47: 42$ & $\mathrm{CA}$ & 45 & 45 & - & & $\mathrm{mP}-22$ \\
\hline B50 & $0: 49: 02$ & $-73: 21: 44$ & c & 33 & 33 & - & 66 & $\mathrm{mT}-8$ \\
\hline BS41,SMC_OGLE194 & $0: 49: 06$ & $-73: 21: 10$ & C & 33 & 33 & - & & $\mathrm{mT}-8$ \\
\hline L39,SMC_OGLE54 & $0: 49: 18$ & $-73: 22: 20$ & C & 42 & 33 & 170 & 66 & $\mathrm{mT}-8$, in BS43 \\
\hline SMC-N33,L61-138,MA 297 & $0: 49: 29$ & $-73: 26: 33$ & $\mathrm{NC}$ & 18 & 15 & 80 & 12 & mP-23, in SMC-DEM44 \\
\hline MA301 & $0: 49: 30$ & $-73: 26: 23$ & $\mathrm{NC}$ & 21 & 18 & 80 & & mP-23,in SMC-DEM44 \\
\hline SMC_OGLE56 & $0: 49: 36$ & $-72: 50: 13$ & $\mathrm{CA}$ & 48 & 36 & 100 & 70 & $\mathrm{mT}-9$, in SMC-DEM46e \\
\hline H86-110 & $0: 49: 44$ & $-72: 51: 14$ & $\mathrm{CA}$ & 45 & 33 & 160 & 70 & $\mathrm{mT}-9$ \\
\hline H86-109,SMC_OGLE58 & $0: 49: 45$ & $-72: 51: 58$ & C & 27 & 27 & - & & $\mathrm{mT}-9$ \\
\hline MA317 & $0: 49: 42$ & $-73: 10: 37$ & $\mathrm{NC}$ & 18 & 15 & 20 & 20 & $\mathrm{mP}-24$ \\
\hline SMC-N34,L61-142,SMC-DEM50, & $0: 49: 46$ & $-73: 10: 25$ & $\mathrm{NC}$ & 39 & 27 & 120 & & $\mathrm{mP}-24 \& \mathrm{MA} 322$ \\
\hline B53,SMC_OGLE197 & $0: 50: 04$ & $-73: 23: 04$ & C & 57 & 57 & - & 80 & $\mathrm{mP}-25$ \\
\hline B55,SMC_OGLE60 & $0: 50: 22$ & $-73: 23: 16$ & C & 42 & 36 & 110 & & $\mathrm{mP}-25$ \\
\hline SMC_OGLE199 & $0: 50: 15$ & $-73: 03: 15$ & $\mathrm{CA}$ & 15 & 15 & - & 35 & $\mathrm{mP}-26$, in? sup? SMC-DEM51 \\
\hline BS45,SMC_OGLE59 & $0: 50: 16$ & $-73: 02: 00$ & $\mathrm{CA}$ & 60 & 54 & 70 & & $\mathrm{mP}-26$, in SMC-DEM51 \\
\hline H86-106w & $0: 50: 31$ & $-73: 20: 11$ & C & 30 & 24 & 90 & 23 & mP-27, in SMC-DEM 52 \\
\hline H86-106е & $0: 50: 37$ & $-73: 20: 11$ & C & 33 & 27 & 80 & & mP-27, in SMC-DEM52 \\
\hline H86-115,SMC_OGLE63 & $0: 50: 37$ & $-73: 03: 28$ & $\mathrm{AC}$ & 96 & 72 & 40 & 77 & mP-28,in SMC-DEM51 \\
\hline SMC_OGLE65 & $0: 50: 55$ & $-73: 03: 27$ & C & 39 & 39 & - & & $\mathrm{mP}-28$ \\
\hline BS46,SMC_OGLE200 & $0: 50: 39$ & $-72: 58: 44$ & c & 30 & 27 & 60 & 47 & $\mathrm{mP}-29$, in L40 \\
\hline H86-116,SMC_OGLE64 & $0: 50: 40$ & $-72: 57: 55$ & C & 30 & 30 & - & & $\mathrm{mP}-29$, in L40 \\
\hline BS48,SMC_OGLE201 & $0: 50: 42$ & $-73: 23: 49$ & $\mathrm{AC}$ & 51 & 33 & 80 & 46 & mP-30, in SMC-DEM53 \\
\hline H86-108,MA401 & $0: 50: 53$ & $-73: 24: 22$ & $\mathrm{NA}$ & 60 & 60 & - & & mP-30,in SMC-DEM 53 \\
\hline B59,L61-183,MA488, & $0: 51: 44$ & $-72: 50: 25$ & $\mathrm{CN}$ & 48 & 36 & 80 & 26 & mP-31 \& SMC_OGLE 73 \\
\hline
\end{tabular}


Table 1. continued

\begin{tabular}{|c|c|c|c|c|c|c|c|c|}
\hline Name & $\begin{array}{r}\mathrm{RA}(2000) \\
\mathrm{h}: \mathrm{m}: \mathrm{s}\end{array}$ & $\begin{array}{c}\operatorname{Dec}(2000) \\
\text { o: ' : } " \text { " }\end{array}$ & $\mathrm{T}$ & $\begin{array}{r}\text { Dmax } \\
\prime \prime\end{array}$ & $\begin{array}{r}\text { Dmin } \\
\prime \prime\end{array}$ & $\begin{array}{r}\mathrm{PA} \\
\mathrm{O}\end{array}$ & $\begin{array}{r}\text { Separ. } \\
\prime \prime\end{array}$ & Comments \\
\hline (1) & (2) & (3) & (4) & (5) & (6) & (7) & (8) & (9) \\
\hline SMC-N46,L61-184,SMC-DEM62, & $0: 51: 47$ & $-72: 50: 47$ & $\mathrm{NC}$ & 39 & 39 & - & & mP-31 \& MA498 \\
\hline H86-120 & $0: 51: 46$ & $-73: 28: 01$ & $\mathrm{C}$ & 21 & 21 & - & 43 & mT-10,in SMC-DEM $70 \mathrm{~s}$ \\
\hline BS53 & $0: 51: 49$ & $-73: 28: 38$ & A & 39 & 33 & 150 & & $\mathrm{mT}-10$, in SMC-DEM $70 \mathrm{~s}$ \\
\hline H86-122 & $0: 51: 58$ & $-73: 27: 41$ & C & 27 & 27 & $:$ & 43 & $\mathrm{mT}-10$, in SMC-DEM $70 \mathrm{~s}$ \\
\hline BS56,SMC_OGLE77 & $0: 52: 13$ & $-73: 00: 12$ & $\mathrm{C}$ & 42 & 33 & 90 & 56 & $\mathrm{mP}-32$ \\
\hline H86-130,SMC_OGLE78 & $0: 52: 17$ & $-73: 01: 04$ & C & 45 & 36 & 0 & & $\mathrm{mP}-32$ \\
\hline B64,SMC_OGLE210 & $0: 52: 30$ & $-73: 02: 59$ & C & 42 & 42 & - & 56 & $\mathrm{mP}-33$, in $\mathrm{H}-\mathrm{A} 29$ \\
\hline BS57,SMC_OGLE211 & $0: 52: 32$ & $-73: 02: 10$ & C & 39 & 27 & 60 & & $\mathrm{mP}-33$, in $\mathrm{H}-\mathrm{A} 29$ \\
\hline H86-134w,SMC_OGLE212 & $0: 52: 45$ & $-72: 59: 24$ & $\mathrm{C}$ & 30 & 30 & - & 19 & $\mathrm{mT}-11$, in $\mathrm{H}-\mathrm{A} 30$ \\
\hline B65,SMC_OGLE83 & $0: 52: 44$ & $-72: 58: 48$ & $\mathrm{C}$ & 45 & 45 & - & & $\mathrm{mT}-11$ \\
\hline H86-134e,SMC_OGLE213 & $0: 52: 48$ & $-72: 59: 22$ & $\mathrm{C}$ & 30 & 27 & 0 & 19 & $\mathrm{mT}-11$,in $\mathrm{H}-\mathrm{A} 30$ \\
\hline BS61 & $0: 52: 43$ & $-73: 01: 45$ & $\mathrm{CA}$ & 36 & 27 & 80 & 42 & $\mathrm{mP}-34$,in $\mathrm{H}-\mathrm{A} 29$ \\
\hline $\mathrm{BS} 255$ & $0: 52: 52$ & $-73: 01: 45$ & $\mathrm{C}$ & 24 & 18 & 70 & & $\mathrm{mP}-34$ \\
\hline BS63,SMC_OGLE84 & $0: 52: 47$ & $-73: 24: 25$ & C & 30 & 24 & 150 & 19 & $\mathrm{mT}-12$, in SMC-DEM 73 \\
\hline B67,SMC_OGLE87 & $0: 52: 49$ & $-73: 24: 43$ & $\mathrm{C}$ & 39 & 30 & 110 & & $\mathrm{mT}-12$, in SMC-DEM 73 \\
\hline NGC $294, \mathrm{~L} 47, \mathrm{ESO} 29 \mathrm{SC} 22$, & $0: 53: 06$ & $-73: 22: 49$ & C & 102 & 102 & - & & mT-12 \& SMC_OGLE90 \\
\hline H86-140,SMC_OGLE214 & $0: 53: 09$ & $-72: 49: 58$ & $\mathrm{C}$ & 27 & 24 & 50 & 15 & mP-35 \\
\hline H86-139 & $0: 53: 11$ & $-72: 50: 05$ & C & 21 & 18 & 40 & & $\mathrm{mP}-35$ \\
\hline BS67 & $0: 53: 32$ & $-73: 21: 03$ & $\mathrm{AC}$ & 39 & 39 & - & 51 & $\mathrm{mP}-36$ \\
\hline BS68,SMC_OGLE95 & $0: 53: 42$ & $-73: 21: 32$ & $\mathrm{CA}$ & 54 & 45 & 130 & & $\mathrm{mP}-36$ \\
\hline $\mathrm{B} 72$ & $0: 53: 26$ & $-72: 40: 57$ & $\mathrm{C}$ & 72 & 72 & - & & $\mathrm{m} 6$, in $\mathrm{H}-\mathrm{A} 35$ \\
\hline H86-143,SMC_OGLE93 & $0: 53: 31$ & $-72: 40: 04$ & $\mathrm{C}$ & 48 & 48 & - & & $\mathrm{m} 6$, in $\mathrm{H}-\mathrm{A} 35$ \\
\hline BS257 & $0: 53: 36$ & $-72: 38: 30$ & $\mathrm{AC}$ & 48 & 39 & 0 & & m6 \\
\hline SMC-N52A,L61-243, & $0: 53: 40$ & $-72: 39: 35$ & $\mathrm{NC}$ & 30 & 30 & - & 20 & m6,in H-A35 \& SMC-DEM77sw,MA696,SMC_OGLE94 \\
\hline SMC-N52B,L61-244,B73, & $0: 53: 42$ & $-72: 39: 15$ & $\mathrm{NC}$ & 30 & 30 & - & 20 & m6,in H-A35 \& SMC-DEM77ne,MA699,SMC_OGLE96 \\
\hline H86-148 & $0: 53: 55$ & $-72: 40: 08$ & $\mathrm{C}$ & 30 & 30 & - & & $\mathrm{m} 6$, in $\mathrm{H}-\mathrm{A} 35$ \\
\hline BS69,SMC_OGLE217 & $0: 53: 56$ & $-72: 51: 24$ & $\mathrm{CA}$ & 36 & 24 & 45 & 75 & $\mathrm{mP}-37$ \\
\hline BS72,SMC_OGLE97 & $0: 54: 11$ & $-72: 51: 54$ & $\mathrm{CA}$ & 45 & 36 & 20 & & $\mathrm{mP}-37$ \\
\hline B78 & $0: 54: 45$ & $-72: 07: 46$ & $\mathrm{C}$ & 66 & 48 & 110 & 56 & $\mathrm{mP}-38$,in $\mathrm{H}-\mathrm{A} 37$ \\
\hline L51,ESO $51 \mathrm{SC} 7$ & $0: 54: 54$ & $-72: 06: 46$ & $\mathrm{C}$ & 60 & 45 & 170 & & $\mathrm{mP}-38$, in $\mathrm{H}-\mathrm{A} 37$ \\
\hline H86-159,SMC_OGLE102 & $0: 55: 12$ & $-72: 41: 00$ & $\mathrm{C}$ & 30 & 24 & 130 & 45 & $\mathrm{mP}-39$,in BS260 \\
\hline H86-160 & $0: 55: 21$ & $-72: 40: 10$ & $\mathrm{C}$ & 24 & 24 & - & & $\mathrm{mP}-39$,in BS260 \\
\hline BS81,SMC_OGLE223 & $0: 56: 26$ & $-72: 29: 45$ & $\mathrm{C}$ & 36 & 33 & 0 & 43 & $\mathrm{mP}-40$, in $\mathrm{H}-\mathrm{A} 40$ \\
\hline H86-172,SMC_OGLE108 & $0: 56: 34$ & $-72: 30: 08$ & $\mathrm{C}$ & 33 & 33 & - & & $\mathrm{mP}-40$, in $\mathrm{H}-\mathrm{A} 40$ \\
\hline H86-175,SMC_OGLE227 & $0: 57: 50$ & $-72: 26: 24$ & $\mathrm{C}$ & 24 & 24 & - & 38 & $\mathrm{mP}-41$ \\
\hline H86-179,SMC_OGLE112 & $0: 57: 57$ & $-72: 26: 42$ & $\mathrm{C}$ & 24 & 24 & - & & $\mathrm{mP}-41$ \\
\hline H86-177,SMC_OGLE226 & $0: 57: 50$ & $-72: 30: 29$ & $\mathrm{C}$ & 45 & 45 & - & 46 & mP-42, in B-OB 13 \\
\hline H86-176 & $0: 57: 53$ & $-72: 29: 48$ & $\mathrm{C}$ & 36 & 30 & 90 & & $\mathrm{mP}-42$ \\
\hline SMC-N62,SMC-DEM93 & $0: 57: 56$ & $-72: 39: 26$ & $\mathrm{NA}$ & 72 & 72 & - & 75 & $\mathrm{mT}-13$, in H-A 42 \\
\hline SMC-N63,L61-331,SMC-DEM94, & $0: 58: 16$ & $-72: 38: 47$ & NA & 36 & 36 & - & & mT-13,in H-A42 \& MA1065,SMC_OGLE113 \\
\hline SMC-N64A,L61-335,SMC-DEM95 & $0: 58: 26$ & $-72: 39: 57$ & $\mathrm{NC}$ & 48 & 39 & 70 & 75 & mT-13,in SMC-N64 \& H86-182,MA1071,SMC_OGLE114 \\
\hline BS269 & $0: 58: 19$ & $-72: 13: 10$ & $\mathrm{CA}$ & 24 & 18 & 60 & 35 & $\mathrm{mP}-43$ \\
\hline BS270 & $0: 58: 23$ & $-72: 12: 43$ & $\mathrm{CA}$ & 33 & 30 & 130 & & $\mathrm{mP}-43$ \\
\hline BS271 & $0: 58: 37$ & $-72: 13: 27$ & $\mathrm{NC}$ & 39 & 30 & 30 & 35 & mP-44,in SMC-DEM98 \\
\hline BS272,SMC_OGLE229 & $0: 58: 38$ & $-72: 14: 04$ & $\mathrm{NC}$ & 39 & 39 & - & & mP-44, in SMC-DEM98 \\
\hline BS93 & $0: 59: 36$ & $-71: 44: 13$ & $\mathrm{C}$ & 24 & 21 & 10 & 17 & mT-14,in SMC-DEM105 \\
\hline в97 & $0: 59: 37$ & $-71: 44: 40$ & $\mathrm{C}$ & 30 & 30 & - & & mT-14,in SMC-DEM105 \\
\hline BS273 & $0: 59: 40$ & $-71: 44: 34$ & $\mathrm{AC}$ & 30 & 27 & 120 & 17 & mT-14,in SMC-DEM105 \\
\hline IC $1611, \mathrm{~K} 40, \mathrm{~L} 61, \mathrm{ESO} 29 \mathrm{SC} 27$, & $0: 59: 48$ & $-72: 20: 02$ & $\mathrm{C}$ & 90 & 90 & - & & m4-1 \& SMC_OGLE118 \\
\hline H86-186,SMC_OGLE119 & $0: 59: 57$ & $-72: 22: 24$ & $\mathrm{C}$ & 36 & 36 & - & 29 & m4-1,att SMC-DEM114 \\
\hline IC $1612, \mathrm{~K} 41, \mathrm{~L} 62, \mathrm{ESO} 29 \mathrm{SC} 28$, & 1:00:01 & $-72: 22: 08$ & $\mathrm{C}$ & 72 & 48 & 20 & 29 & m4-1,att SMC-DEM114 \& SMC_OGLE120 \\
\hline K42,L63,SMC_OGLE124 & $1: 00: 34$ & $-72: 21: 56$ & $\mathrm{C}$ & 51 & 51 & - & & m4-1, att SMC-DEM114 \\
\hline B98sw & $1: 00: 21$ & $-73: 52: 51$ & $\mathrm{C}$ & 36 & 27 & 40 & 33 & $\mathrm{mP}-45$ \\
\hline B98ne & $1: 00: 28$ & $-73: 52: 32$ & $\mathrm{C}$ & 33 & 27 & 130 & & $\mathrm{mP}-45$ \\
\hline H86-189,SMC_OGLE123 & $1: 00: 33$ & $-72: 14: 23$ & $\mathrm{C}$ & 24 & 24 & - & 68 & $\mathrm{mP}-46$ \\
\hline H86-190,SMC_OGLE230 & $1: 00: 33$ & $-72: 15: 31$ & $\mathrm{C}$ & 24 & 24 & - & & $\mathrm{mP}-46$ \\
\hline H86-191,SMC_OGLE231 & $1: 00: 58$ & $-72: 32: 25$ & $\mathrm{C}$ & 48 & 48 & - & 81 & mP-47,in? SMC-DEM114 \\
\hline H86-194,SMC_OGLE232 & $1: 01: 14$ & $-72: 33: 03$ & $\mathrm{C}$ & 51 & 51 & - & & mP-47,in? SMC-DEM114 \\
\hline BS102 & $1: 01: 14$ & $-73: 47: 45$ & $\mathrm{C}$ & 30 & 21 & 100 & 42 & $\mathrm{mP}-48$ \\
\hline HW44 & $1: 01: 22$ & $-73: 47: 15$ & $\mathrm{C}$ & 45 & 45 & - & & $\mathrm{mP}-48$ \\
\hline B110 & $1: 02: 11$ & $-72: 00: 11$ & $\mathrm{C}$ & 33 & 27 & 70 & 65 & $\mathrm{mP}-49, \mathrm{br}^{*}$ in, in $\mathrm{H}-\mathrm{A} 49$ \\
\hline B112 & $1: 02: 23$ & $-72: 00: 11$ & $\mathrm{C}$ & 51 & 45 & 60 & & $\mathrm{mP}-49, \mathrm{br}^{*}$ in, in $\mathrm{H}-\mathrm{A} 49$ \\
\hline $\mathrm{K} 45 \mathrm{w}, \mathrm{L} 69 \mathrm{w}$ & $1: 02: 45$ & $-73: 44: 19$ & $\mathrm{C}$ & 33 & 27 & 40 & 47 & $\mathrm{mP}-50$ \\
\hline $\mathrm{K} 45 \mathrm{e}, \mathrm{L} 69 \mathrm{w}$ & $1: 02: 49$ & $-73: 44: 25$ & $\mathrm{C}$ & 24 & 24 & - & & $\mathrm{mP}-50$ \\
\hline NGC $376, K 49, \mathrm{~L} 72$,ESO $29 \mathrm{SC} 29$, & $1: 03: 53$ & $-72: 49: 34$ & $\mathrm{C}$ & 108 & 108 & - & 75 & mP-51 \& SMC_OGLE139 \\
\hline BS114,SMC_OGLE235 & 1:03:59 & $-72: 48: 18$ & $\mathrm{AC}$ & 42 & 30 & 110 & & mP-51 \\
\hline SMC_OGLE138 & $1: 03: 53$ & $-72: 06: 11$ & $\mathrm{CA}$ & 36 & 36 & - & 82 & $\mathrm{mP}-52$, in $\mathrm{H}-\mathrm{A} 53$ \\
\hline SMC_OGLE144,SMC_OGLE236 & $1: 04: 05$ & $-72: 07: 15$ & $\mathrm{CA}$ & 36 & 36 & - & & $\mathrm{mP}-52$ \\
\hline BS122 & $1: 04: 18$ & $-73: 10: 21$ & $\mathrm{C}$ & 21 & 21 & - & 26 & $\mathrm{mP}-53$ \\
\hline B119 & 1:04:19 & $-73: 09: 54$ & $\mathrm{C}$ & 36 & 36 & - & & $\mathrm{mP}-53$ \\
\hline SMC-N78A,L61-438,MA1512 & 1:05:04 & $-71: 59: 01$ & NC & 24 & 21 & 140 & 23 & $\mathrm{~m} 5$, in NGC 395 \\
\hline SMC-N78B,L61-439, & 1:05:04 & $-71: 59: 25$ & NC & 24 & 18 & 100 & & m5,in NGC 395 \& MA1508/1514,SMC_OGLE145 \\
\hline MA1520,SMC_OGLE147 & 1:05:08 & $-71: 59: 45$ & NC & 30 & 27 & 130 & 23 & $\mathrm{~m} 5$, in NGC 395 \\
\hline SMC-N78D,SMC-DEM127 & $1: 05: 11$ & $-71: 58: 28$ & NA & 54 & 54 & - & & $\mathrm{m} 5$,att SMC-N78 \\
\hline SMC_OGLE146 & 1:05:13 & -715942 & $\mathrm{NA}$ & 27 & 27 & - & & m5, in NGC 395 \\
\hline BS130 & 1:05:56 & $-72: 04: 11$ & A & 57 & 36 & 140 & 30 & mP-54,att SMC-N78 \\
\hline $\mathrm{BS} 132$ & 1:06:01 & $-72: 03: 37$ & $\mathrm{CA}$ & 42 & 33 & 150 & & mP-54,att SMC-N78 \\
\hline BS133 & $1: 06: 23$ & $-71: 55: 12$ & A & 39 & 30 & 30 & & $\mathrm{mT}-15$,in BS134 \\
\hline BS135 & 1:06:40 & $-71: 55: 13$ & $\mathrm{CA}$ & 39 & 39 & - & 47 & $\mathrm{mT}-15$, in BS 134 \\
\hline BS136 & $1: 06: 48$ & $-71: 54: 55$ & $\mathrm{CA}$ & 42 & 42 & - & 47 & $\mathrm{mT}-15$, in SMC-DEM132 \\
\hline B134 & 1:09:01 & $-73: 12: 24$ & $\mathrm{CA}$ & 48 & 33 & 80 & & $\mathrm{~m} 4-2$, in $\mathrm{H}-\mathrm{A} 60$ \\
\hline BS142 & 1:09:07 & $-73: 12: 01$ & $\mathrm{C}$ & 24 & 21 & 70 & 31 & $\mathrm{~m} 4-2$, in $\mathrm{H}-\mathrm{A} 60$ \\
\hline IC 1644, SMC-N81,L61-481, & $1: 09: 12$ & $-73: 11: 42$ & $\mathrm{NC}$ & 48 & 39 & 40 & 31 & m4-2,in H-A60 \& ESO 29EN35,MA1688/1687 \\
\hline B135 & 1:09:19 & $-73: 11: 15$ & $\mathrm{C}$ & 33 & 24 & 60 & & $\mathrm{~m} 4-2$, in $\mathrm{H}-\mathrm{A} 60$ \\
\hline $\mathrm{NGC} 422, \mathrm{~K} 62, \mathrm{~L} 87, \mathrm{ESO} 51 \mathrm{SC} 22$ & $1: 09: 25$ & $-71: 46: 00$ & $\mathrm{C}$ & 60 & 60 & - & 64 & mP-55 \\
\hline IC $1641, \mathrm{HW} 62, \mathrm{ESO} 51 \mathrm{SC} 21$ & 1:09:39 & $-71: 46: 07$ & $\mathrm{C}$ & 45 & 39 & 40 & & $\mathrm{mP}-55$ \\
\hline HW71nw & $1: 15: 30$ & $-72: 22: 36$ & $\mathrm{C}$ & 18 & 18 & - & 17 & $\mathrm{mP}-56$ \\
\hline HW71se & $1: 15: 33$ & $-72: 22: 50$ & $\mathrm{C}$ & 39 & 33 & 170 & & $\mathrm{mP}-56$ \\
\hline
\end{tabular}


Table 2. Age, reddening and morphological classification

\begin{tabular}{|c|c|c|c|c|c|c|}
\hline $\begin{array}{l}\text { Name } \\
\text { (1) }\end{array}$ & $\begin{array}{r}\text { Age } \\
\text { Myr } \\
(2)\end{array}$ & $\begin{array}{r}E(B-V) \\
(3)\end{array}$ & $\begin{array}{r}\text { Age OGLE } \\
\text { Myr } \\
(4)\end{array}$ & $\begin{array}{r}E(B-V)_{\mathrm{OGLE}} \\
(5)\end{array}$ & $\begin{array}{r}\text { Morphologies } \\
(6)\end{array}$ & $\begin{array}{l}\text { Comments } \\
(7)\end{array}$ \\
\hline BS3 & & & & & $\frac{b}{b}$ & mT-1 outside OGLEII \\
\hline H86-2 & & & & & $\mathrm{b}$ & mT-1 outside OGLEII \\
\hline BS4 & & & & & & mT- 1 outside OGLEII \\
\hline H86-38 & $251 \pm 25$ & 0.10 & & & & $\mathrm{mP}-5$ \\
\hline BS10 & $>560$ & 0.10 & & & & $\mathrm{mP}-5$ \\
\hline B15 & $>1000$ & 0.07 & & & & $\mathrm{mP}-8$ \\
\hline H86-57 & $>1000$ & 0.07 & & & & $\mathrm{mP}-8$ \\
\hline NGC 220,SMC_OGLE8 & $65 \pm 13$ & 0.07 & $100 \pm 23$ & 0.07 & $\mathrm{~d}$ & $\mathrm{mT}-2$ \\
\hline NGC 222,SMC_OGLE9 & $70 \pm 7$ & 0.07 & $100 \pm 23$ & 0.07 & d & $\mathrm{mT}-2$ \\
\hline B23,SMC_OGLE170 & $<100$ & 0.07 & & & d & $\mathrm{mT}-2$ \\
\hline NGC 231,SMC_OGLE11 & $65 \pm 8$ & 0.12 & $79 \pm 18$ & 0.08 & & $\mathrm{mP}-10$ \\
\hline BS15 & $70 \pm 7$ & 0.10 & & & & $\mathrm{mP}-10$ \\
\hline B29 & $150 \pm 70$ & 0.10 & & & & $\mathrm{mP}-12$ \\
\hline HW16,SMC_OGLE13 & $<20$ & 0.07 & $20 \pm 15$ & 0.05 & & $\mathrm{mP}-12$ \\
\hline NGC 241,SMC_OGLE17 & $55 \pm 5$ & 0.12 & $79 \pm 18$ & 0.10 & $\mathrm{~b}$ & $\mathrm{mP}-13$ \\
\hline NGC 242,SMC_OGLE18 & $65 \pm 10$ & 0.10 & $79 \pm 18$ & 0.10 & $\mathrm{~b}$ & $\mathrm{mP}-13$ \\
\hline B31,SMC_OGLE19,SMC_OGLE175 & $280 \pm 30$ & 0.10 & $400 \pm 92$ & 0.08 & & $\mathrm{mT}-4$ \\
\hline BS20,SMC_OGLE20 & $450 \pm 50$ & 0.08 & $400 \pm 92$ & 0.08 & & $\mathrm{mT}-4$ \\
\hline H86-70,SMC_OGLE21 & $450 \pm 50$ & 0.07 & & & & $\mathrm{mT}-4$ \\
\hline BS27,SMC_OGLE177 & $<25$ & 0.18 & $79 \pm 37$ & 0.08 & & $\mathrm{mP}-14$ \\
\hline SMC-N10 & HII Region & & & & & $\mathrm{mP}-14$ \\
\hline NGC 248n,SMC_OGLE26n & HII Region & & & & & $\mathrm{mP}-15$ \\
\hline NGC 248s,SMC_OGLE26s & HII Region & & & & & $\mathrm{mP}-15$ \\
\hline 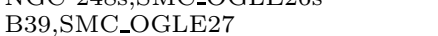 & $450 \pm 50$ & 0.07 & & & $\mathrm{~b}$ & $\mathrm{mP}-16$ \\
\hline BS30 & $450 \pm 50$ & 0.07 & & & $\mathrm{~b}$ & $\mathrm{mP}-16$ \\
\hline B36 & $315 \pm 50$ & 0.03 & & & & $\mathrm{mP}-17$ \\
\hline SMC_OGLE31 & $450 \pm 50$ & 0.03 & & & & $\mathrm{mP}-17$ \\
\hline H86-76,SMC_OGLE182 & $200 \pm 20$ & 0.14 & & & & $\mathrm{mT}-5$ \\
\hline H86-78n,SMC_OGLE33n & $<25$ & 0.14 & $16 \pm 9$ & 0.15 & & $\mathrm{mT}-5$ \\
\hline H86-78s,SMC_OGLE33s & $<30$ & 0.14 & $16 \pm 9$ & 0.15 & & $\mathrm{mT}-5$ \\
\hline L31,SMC_OGLE36 & $250 \pm 50$ & 0.10 & & & & $\mathrm{mT}-6$ \\
\hline H86-83,SMC_OGLE35 & $180 \pm 50$ & 0.10 & & & & $\mathrm{mT}-6$ \\
\hline H86-84,SMC_OGLE185 & $250 \pm 50$ & 0.10 & & & & $\mathrm{mT}-6$ \\
\hline H86-86,SMC_OGLE40 & $350 \pm 50$ & 0.03 & & & & $\mathrm{mP}-19$ \\
\hline H86-87,SMC_OGLE187 & $140 \pm 20$ & 0.07 & $158 \pm 36$ & 0.04 & & $\mathrm{mP}-19$ \\
\hline BS35,SMC_OGLE42 & $400 \pm 100$ & 0.03 & & & & $\mathrm{mP}-21$ \\
\hline K25,SMC_OGLE45 & $250 \pm 50$ & 0.07 & $250 \pm 58$ & 0.07 & & $\mathrm{mP}-21$ \\
\hline MA205 & HII Region & & & & $\mathrm{b}$ & $\mathrm{mT}-7$ \\
\hline SMC-N25,SMC_OGLE189 & HII Region & & & & $\mathrm{b}$ & $\mathrm{mT}-7$ \\
\hline SMC-N26 & HII Region & & & & & $\mathrm{mT}-7$ \\
\hline H86-99,SMC_OGLE190 & $225 \pm 50$ & 0.14 & & & $\mathrm{~d}$ & $\mathrm{mP}-22$ \\
\hline H86-100,SMC_OGLE191 & $200 \pm 50$ & 0.14 & & & d & $\mathrm{mP}-22$ \\
\hline B50 & $<30$ & 0.10 & & & & $\mathrm{mT}-8$ \\
\hline BS41,SMC_OGLE194 & $70 \pm 30$ & 0.10 & $79 \pm 18$ & 0.07 & & $\mathrm{mT}-8$ \\
\hline L39,SMC_OGLE54 & $80 \pm 20$ & 0.10 & $100 \pm 23$ & 0.10 & & $\mathrm{mT}-8$ \\
\hline SMC-N33 & HII Region & & & & $\mathrm{e}$ & $\mathrm{mP}-23$ \\
\hline MA301 & HII Region & & & & $\mathrm{e}$ & $\mathrm{mP}-23$ \\
\hline SMC_OGLE56 & $115 \pm 15$ & 0.18 & & & & $\mathrm{mT}-9$ \\
\hline H86-110 & $<20$ & 0.18 & & & & mT-9 \\
\hline H86-109,SMC_OGLE58 & $180 \pm 20$ & 0.18 & $200 \pm 45$ & 0.17 & & mT-9 \\
\hline MA317 & HII Region & & & & $\mathrm{b}$ & $\mathrm{mP}-24$ \\
\hline SMC-N34 & HII Region & & & & $\mathrm{b}$ & $\mathrm{mP}-24$ \\
\hline B53,SMC_OGLE197 & $200 \pm 50$ & 0.07 & $250 \pm 55$ & 0.08 & & $\mathrm{mP}-25$ \\
\hline B55,SMC_OGLE60 & $160 \pm 30$ & 0.07 & $250 \pm 55$ & 0.08 & & $\mathrm{mP}-25$ \\
\hline SMC_OGLE199 & & & & & & $\mathrm{mP}-26$ \\
\hline BS45,SMC_OGLE59 & $65 \pm 30$ & 0.10 & $63 \pm 14$ & 0.10 & & $\mathrm{mP}-26$ \\
\hline H86-106w & $200 \pm 50$ & 0.10 & & & $\mathrm{e}$ & $\mathrm{mP}-27$ \\
\hline H86-106e & $<30 / 200 \pm 50^{1}$ & $0.12 / 0.10$ & & & $\mathrm{e}$ & $\mathrm{mP}-27$ \\
\hline H86-115,SMC_OGLE63 & & & & & & $\mathrm{mP}-28$ \\
\hline SMC_OGLE65 & $200 \pm 20$ & 0.10 & & & & $\mathrm{mP}-28$ \\
\hline BS46,SMC_OGLE200 & $80 \pm 10$ & 0.07 & $100 \pm 23$ & 0.06 & $\mathrm{~b}$ & $\mathrm{mP}-29$ \\
\hline H86-116,SMC_OGLE64 & $125 \pm 15$ & 0.10 & $126 \pm 29$ & 0.10 & $\mathrm{~b}$ & $\mathrm{mP}-29$ \\
\hline BS48,SMC_OGLE201 & $160 \pm 20$ & 0.10 & & & & $\mathrm{mP}-30$ \\
\hline H86-108,MA401 & $<30$ & 0.12 & & & & $\mathrm{mP}-30$ \\
\hline B59,SMC_OGLE73 & $100 \pm 15$ & 0.14 & $158 \pm 75$ & 0.11 & $\mathrm{e}$ & $\mathrm{mP}-31$ \\
\hline SMC-N46 & HII Region & & & & $\mathrm{e}$ & $\mathrm{mP}-31$ \\
\hline BS56,SMC_OGLE77 & $140 \pm 20$ & 0.07 & $79 \pm 38$ & 0.08 & $\mathrm{~d}$ & $\mathrm{mP}-32$ \\
\hline H86-130,SMC_OGLE78 & $65 \pm 8$ & 0.12 & $79 \pm 18$ & 0.08 & d & $\mathrm{mP}-32$ \\
\hline B64,SMC_OGLE210 & $<10$ & 0.12 & $158 \pm 75$ & 0.07 & & $\mathrm{mP}-33$ \\
\hline BS57,SMC_OGLE211 & $<10$ & 0.12 & & & & $\mathrm{mP}-33$ \\
\hline H86-134w,SMC_OGLE212 & - & - & & & & $\mathrm{mT}-11$ \\
\hline B65,SMC_OGLE83 & $65 \pm 10$ & 0.10 & $63 \pm 30$ & 0.09 & & $\mathrm{mT}-11$ \\
\hline H86-134e,SMC_OGLE213 & - & - & & & & $\mathrm{mT}-11$ \\
\hline
\end{tabular}


Table 2. continued

\begin{tabular}{|c|c|c|c|c|c|c|}
\hline $\begin{array}{l}\text { Name } \\
\text { (1) }\end{array}$ & $\begin{array}{r}\text { Age } \\
\text { Myr } \\
(2)\end{array}$ & $\begin{array}{r}E(B-V) \\
(3)\end{array}$ & $\begin{array}{r}\text { Age }_{\text {OGLE }} \\
\text { Myr } \\
(4)\end{array}$ & $\begin{array}{r}E(B-V)_{\mathrm{OGLE}} \\
(5)\end{array}$ & $\begin{array}{r}\text { Morphologies } \\
(6)\end{array}$ & $\begin{array}{l}\text { Comments } \\
\text { (7) }\end{array}$ \\
\hline BS61 & $250 \pm 50$ & 0.03 & & & & $\mathrm{mP}-34$ \\
\hline BS255 & - & - & & & & $\mathrm{mP}-34$ \\
\hline BS63,SMC_OGLE84 & $450 \pm 50$ & 0.10 & & & d & mT-12 \\
\hline B67,SMC_OGLE87 & $450 \pm 50$ & 0.10 & $500 \pm 115$ & 0.10 & d & $\mathrm{mT}-12$ \\
\hline NGC 294,SMC_OGLE90 & $300 \pm 50$ & 0.10 & $316 \pm 73$ & 0.11 & d & $\mathrm{mT}-12$ \\
\hline H86-140,SMC_OGLE214 & $55 \pm 25$ & 0.10 & & & b & $\mathrm{mP}-35$ \\
\hline H86-139 & $<30$ & 0.10 & & & $\mathrm{~b}$ & $\mathrm{mP}-35$ \\
\hline BS67 & $>300$ & 0.07 & & & & $\mathrm{mP}-36$ \\
\hline BS68,SMC_OGLE95 & $500 \pm 100$ & 0.07 & & & & $\mathrm{mP}-36$ \\
\hline B72 & $80 \pm 10$ & 0.10 & & & & $\mathrm{~m} 6$ \\
\hline H86-143,SMC_OGLE93 & $200 \pm 25$ & 0.10 & & & & $\mathrm{~m} 6$ \\
\hline BS257 & $50 \pm 30^{1}$ & 0.10 & & & & $\mathrm{~m} 6$ \\
\hline SMC-N52A,SMC_OGLE94 & HII Region & & & & & $\mathrm{m} 6$ \\
\hline SMC-N52B,SMC_OGLE96 & HII Region & & & & & $\mathrm{m} 6$ \\
\hline H86-148 & $400 \pm 100$ & 0.10 & & & & $\mathrm{~m} 6$ \\
\hline H86-159,SMC_OGLE102 & $500 \pm 50$ & 0.10 & & & & $\mathrm{mP}-39$ \\
\hline H86-160 & & - & & & & $\mathrm{mP}-39$ \\
\hline BS81,SMC_OGLE223 & $80 \pm 20 / 250 \pm 50^{1}$ & 0.10 & & & & $\mathrm{mP}-40$ \\
\hline H86-172,SMC_OGLE108 & $280 \pm 30$ & 0.07 & & & & $\mathrm{mP}-40$ \\
\hline H86-175,SMC_OGLE227 & $30 \pm 10$ & 0.10 & & & & $\mathrm{mP}-41$ \\
\hline H86-179,SMC_OGLE112 & $<30$ & 0.10 & $32 \pm 23$ & 0.11 & & $\mathrm{mP}-41$ \\
\hline H86-177,SMC_OGLE226 & $<30$ & 0.10 & & & $\mathrm{~d}$ & $\mathrm{mP}-42$ \\
\hline $\mathrm{H} 86-176$ & $<30$ & 0.10 & & & d & $\mathrm{mP}-42$ \\
\hline SMC-N62 & HII Region & & & & & $\mathrm{mT}-13$ \\
\hline SMC-N63,SMC_OGLE113 & HII Region & & & & & $\mathrm{mT}-13$ \\
\hline SMC-N64A,SMC_OGLE114 & HII Region & & & & & $\mathrm{mT}-13$ \\
\hline BS269 & & - & & & & $\mathrm{mP}-43$ \\
\hline BS270 & $<30$ & 0.10 & & & & $\mathrm{mP}-43$ \\
\hline BS271 & $<30$ & 0.12 & & & & $\mathrm{mP}-44$ \\
\hline BS272,SMC_OGLE229 & $<30$ & 0.08 & $79 \pm 18$ & 0.05 & & $\mathrm{mP}-44$ \\
\hline IC1611,SMC_OGLE118 & $100 \pm 20$ & 0.07 & $160 \pm 37$ & 0.08 & & $\mathrm{~m} 4-1$ \\
\hline H86-186,SMC_OGLE119 & $180 \pm 20$ & 0.07 & & & $\mathrm{e}$ & $\mathrm{m} 4-1$ \\
\hline IC1612,SMC_OGLE120 & $100 \pm 50$ & 0.10 & $50 \pm 24$ & 0.07 & $\mathrm{e}$ & $\mathrm{m} 4-1$ \\
\hline K42,SMC_OGLE124 & $20 \pm 10$ & 0.10 & $40 \pm 9$ & 0.06 & & $\mathrm{~m} 4-1$ \\
\hline H86-189,SMC_OGLE123 & $570 \pm 70$ & 0.07 & & & & $\mathrm{mP}-46$ \\
\hline H86-190,SMC_OGLE230 & $125 \pm 13$ & 0.07 & $32 \pm 23$ & 0.08 & & $\mathrm{mP}-46$ \\
\hline H86-191,SMC_OGLE231 & $200 \pm 25$ & 0.07 & & & & $\mathrm{mP}-47$ \\
\hline H86-194,SMC_OGLE232 & $200 \pm 25$ & 0.07 & & & & $\mathrm{mP}-47$ \\
\hline NGC 376,SMC_OGLE139 & $20 \pm 2$ & 0.10 & $32 \pm 7$ & 0.07 & $\mathrm{e}$ & $\mathrm{mP}-51$ \\
\hline BS114,SMC_OGLE235 & $250 \pm 25$ & 0.04 & & & $\mathrm{e}$ & $\mathrm{mP}-51$ \\
\hline SMC_OGLE138 & $<30$ & 0.04 & $25 \pm 20$ & 0.04 & & $\mathrm{mP}-52$ \\
\hline SMC_OGLE144,SMC_OGLE236 & $<30$ & 0.07 & $40 \pm 19$ & 0.05 & & $\mathrm{mP}-52$ \\
\hline SMC-N78A & HII Region & & & & & m5 \\
\hline SMC-N78B,SMC_OGLE145 & HII Region & & $79 \pm 37$ & 0.07 & & m5 \\
\hline MA1520,SMC_OGLE147 & HII Region & & $12 \pm 9$ & 0.06 & & m5 \\
\hline SMC-N78D & HII Region & & & & & $\mathrm{m} 5$ \\
\hline SMC_OGLE146 & HII Region & & $20 \pm 15$ & 0.06 & & $\mathrm{~m} 5$ \\
\hline BS130 & $80 \pm 10$ & 0.10 & & & & $\mathrm{mP}-54$ \\
\hline BS132 & $<30 / 200 \pm 25^{1,2}$ & 0.10 & & & & $\mathrm{mP}-54$ \\
\hline HW71nw & & & & & $\mathrm{e}$ & mP-56 outside OGLEII \\
\hline HW71se & & & & & $\mathrm{e}$ & mP-56 outside OGLEII \\
\hline
\end{tabular}

Notes to Table 2: 1- Age depends on membership of bright stars; 2- Upper main sequence is possibly underpopulated.

imply a common outer isophotal, however the former includes a dimension which is considerably smaller than the smaller cluster diameter. The pair B59/SMC-N46 would be an envelope limiting case (Fig. 4).

As examples, the pair H86-186/IC 1612 (Fig. 5) is embedded in the same isophotal envelope and it is classified as e. The pair H86-140/H86-139 (Fig. 4) shows a bridge linking its members and is classificated as b. In Fig. 6 the pair NGC 376/BS114 shows isophotal distortions, however the outer isophotes of the components are not connected and it is thus classified as $\mathbf{d}$.

We detected relevant isophotal features for about $25 \%$ of the sample distributed as follows: 6 candidate cluster systems with common envelope, 7 with bridge and 5 detached cases.
High isophotal densities such as those observed for B78/L51 (Fig. 5) are related to the high surface brightness which often occurs in blue clusters.

It is important to note that non relevant isophotal features can appear in the maps caused by bright stars or background fluctuations. By means of previous inspections in the original images we verified the non relevant objects. For example, the compact object in the lower right corner of the B39/BS30 isophotal map (Fig. 2) is a bright star, however the compact one to the right of the main cluster can be a star or a compact cluster, as seen in the original form. Higher resolution images would be necessary to check the presence of a third cluster. 

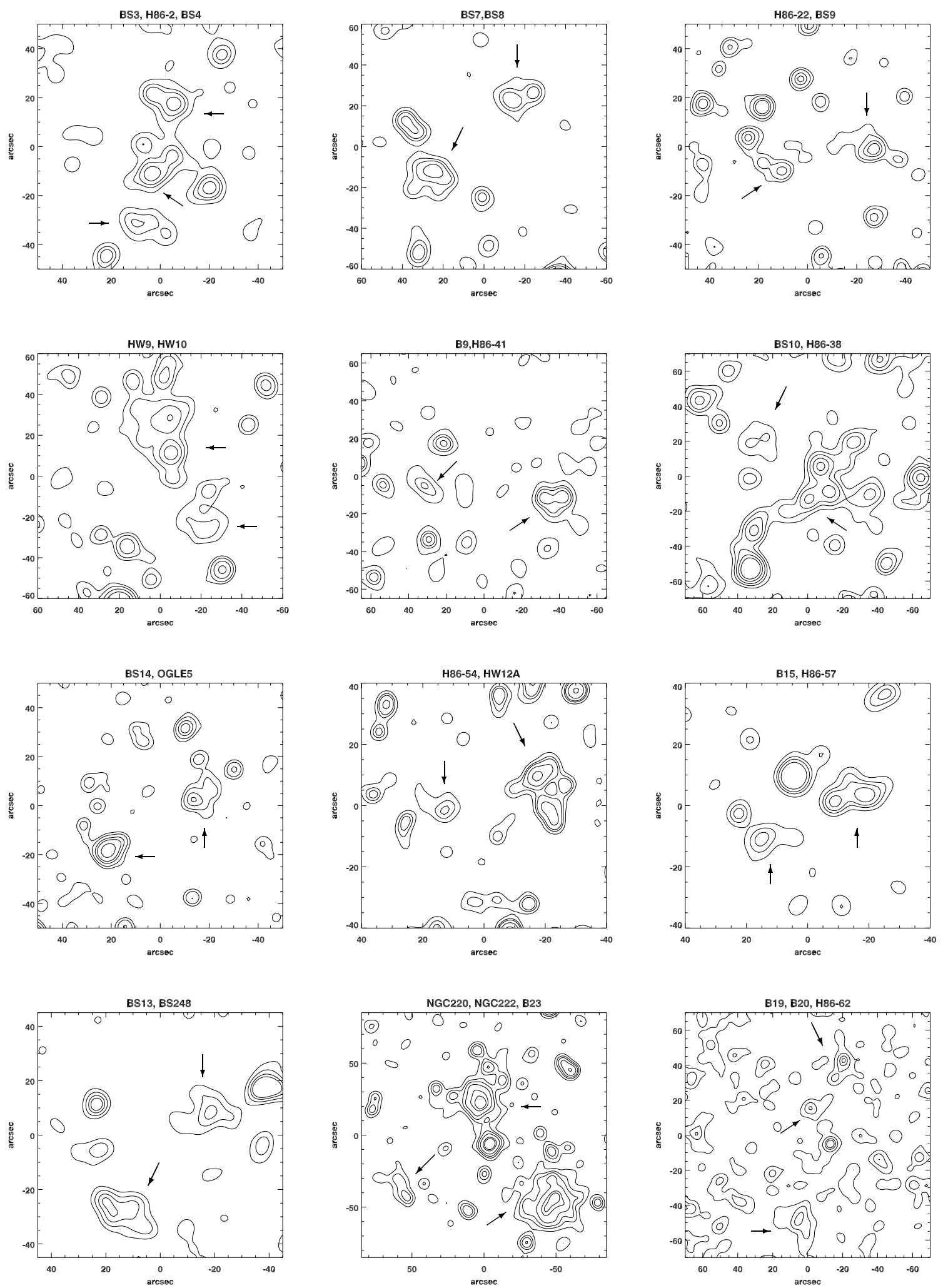

Fig. 1. Isophotal atlas of SMC cluster pairs and multiplets 



Fig. 2. Isophotal atlas of SMC cluster pairs and multiplets 

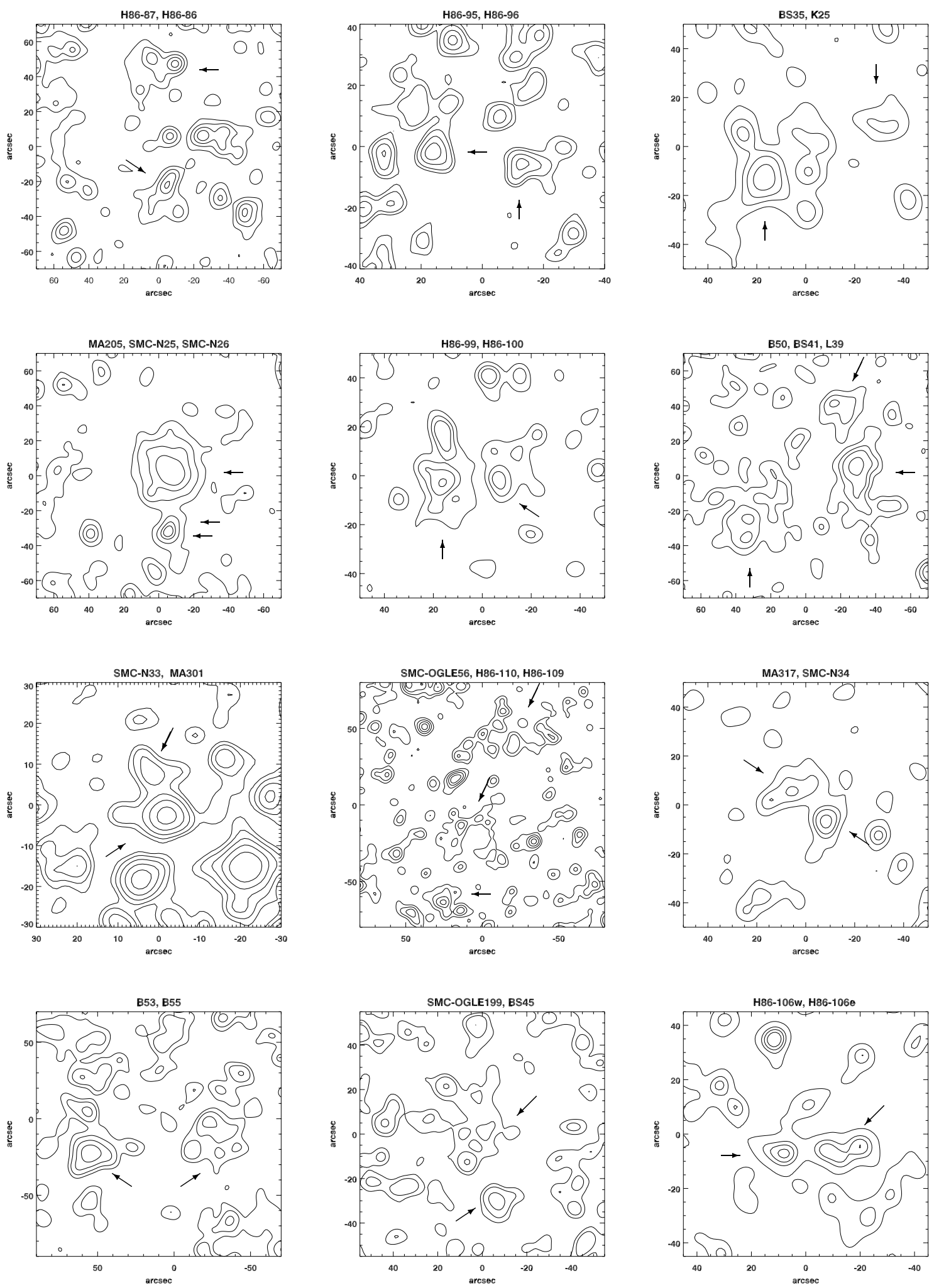

Fig. 3. Isophotal atlas of SMC cluster pairs and multiplets 

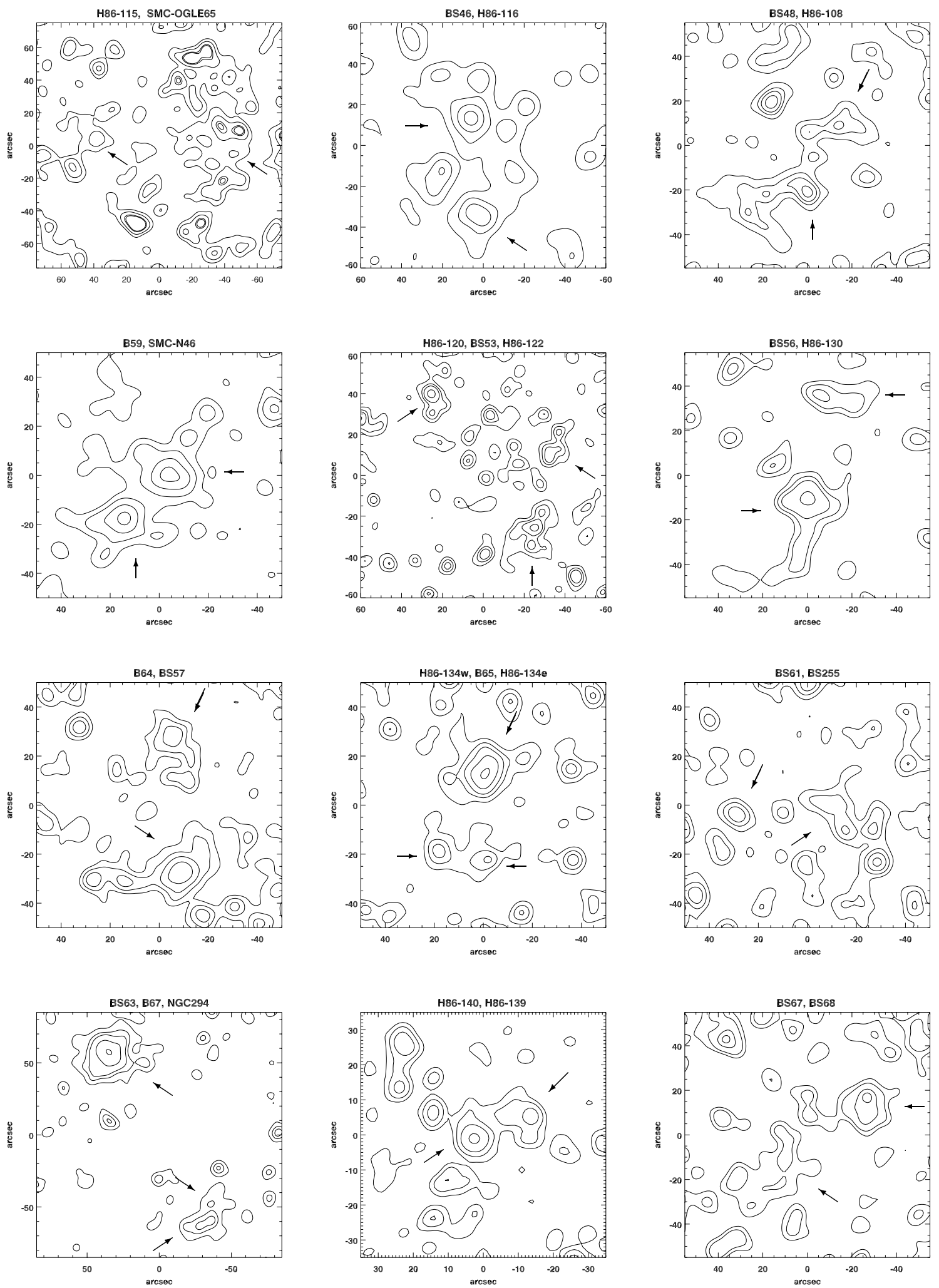

Fig. 4. Isophotal atlas of SMC cluster pairs and multiplets 

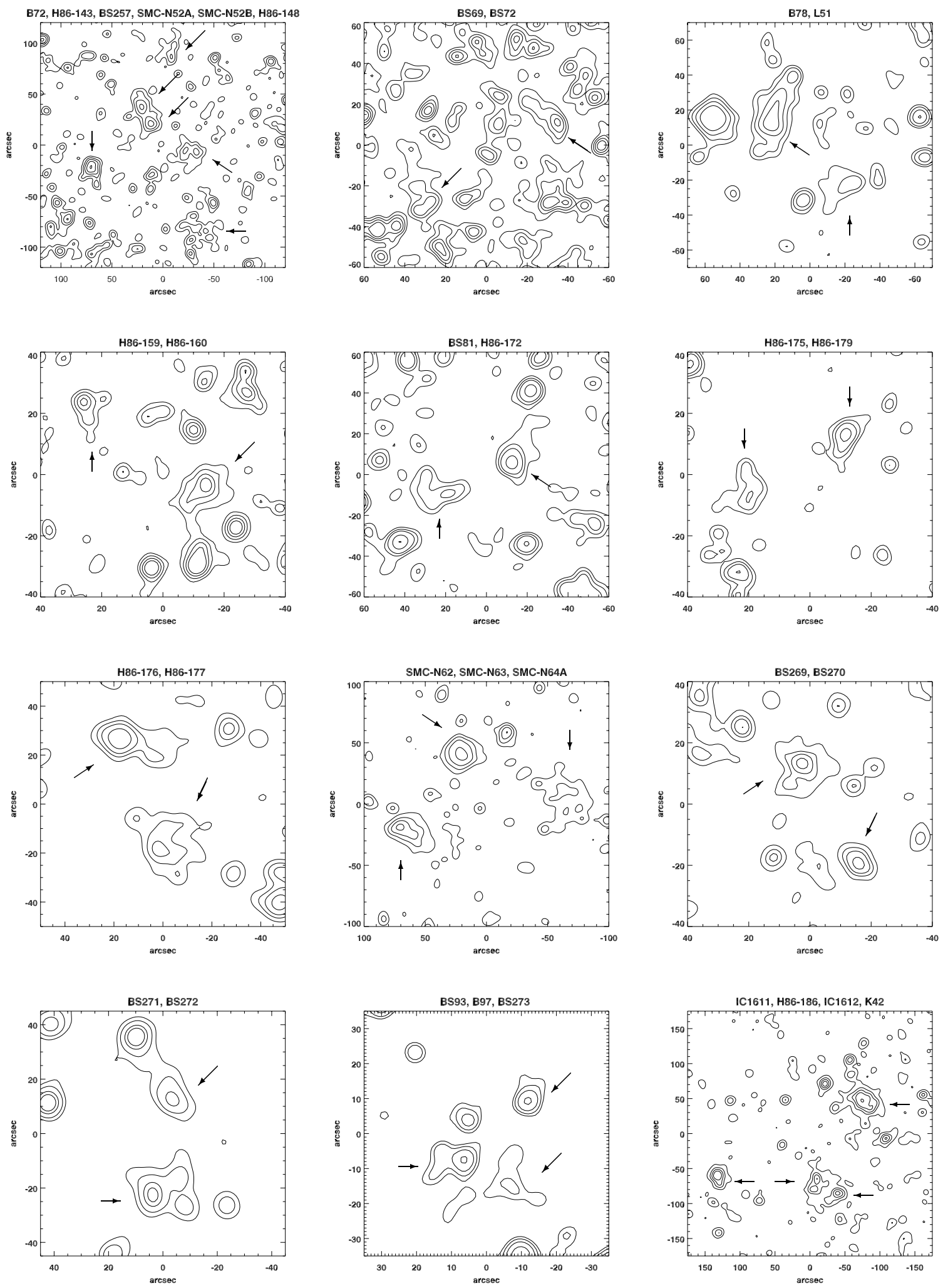

Fig. 5. Isophotal atlas of SMC cluster pairs and multiplets 



Fig. 6. Isophotal atlas of SMC cluster pairs and multiplets 

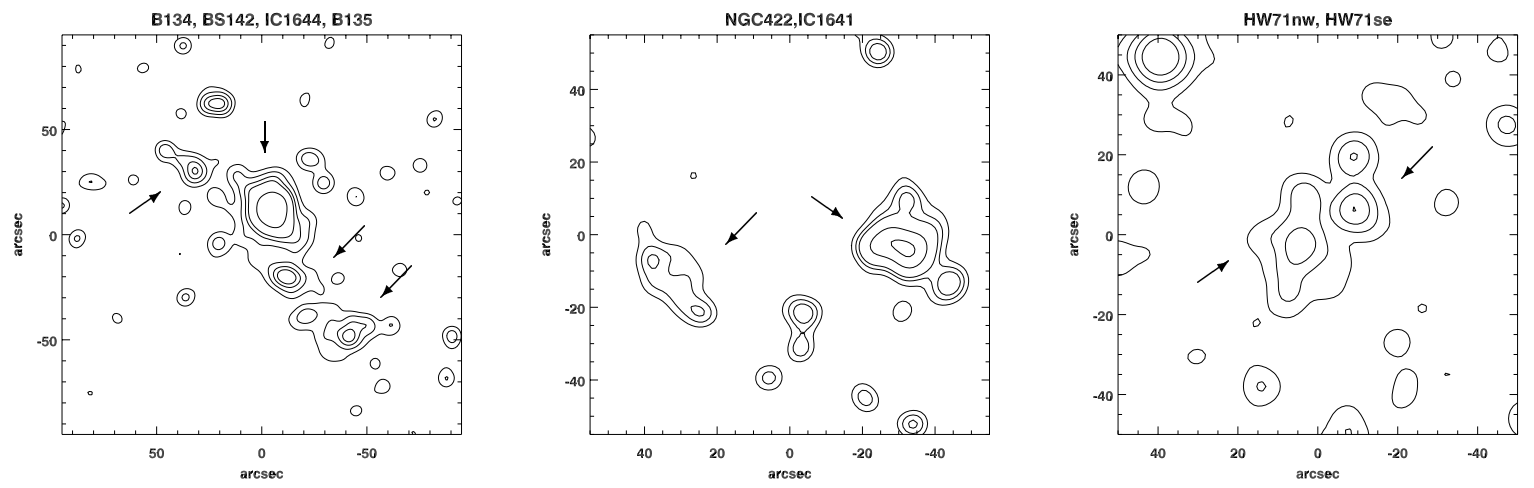

Fig. 7. Isophotal atlas of SMC cluster pairs and multiplets

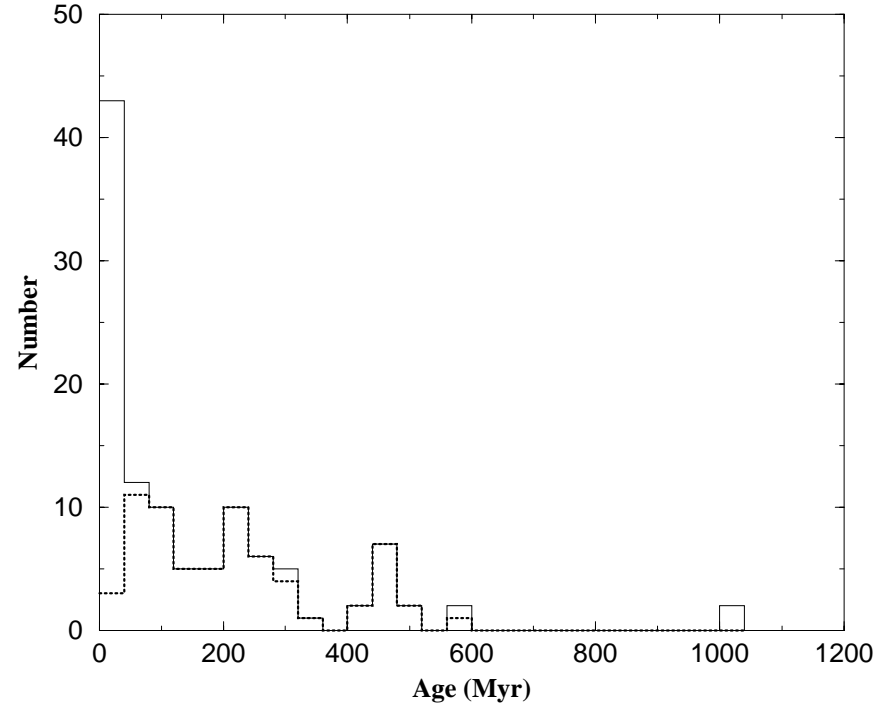

Fig. 8. Age histograms: dotted lines represent clusters with age via CMDs; solid lines are for the same sample as above plus embedded clusters in HII regions, assuming an age 3 Myr. In the latter histogram lower or upper limits in Table 2 were also included by adopting them as cluster ages

\section{Ages of cluster members}

Udalski et al. (1998) provided a BVI photometric survey of the SMC central $\approx 2.4$ square degrees with results for about 2.2 million stars. The data were collected during the OGLE-II microlensing search project (Udalski et al. 1997).

Pietrzyǹski \& Udalski (1999b) studied colourmagnitude diagrams (CMDs) extracted from the BVI database for 93 SMC star clusters, taking into account neighbouring field extractions for comparisons. They estimated reddening values for each cluster using: (i) the mean $I$ band magnitude of red clump stars in the cluster neighbourhood; (ii) the assumed extinction-free magnitude of the SMC's red clump stars, $I=18.34$ mag (Udalski 1998). They derived ages using Bertelli et al.'s (1994) isochrones, adopting as a rule for the SMC a



Fig. 9. Angular distribution of the 176 objects in SMC cluster pairs and multiplets together with HI contours 100, 200, 400 and 600 in units $10^{19}$ atoms $\mathrm{cm}^{-2}$ from Mathewson \& Ford (1984)

metallicity $Z=0.004$, and an absolute distance modulus $(m-M)=18.65$.

Considering the OGLE-II angular coverage we conclude that out of the present 176 objects 133 are therein included. We obtained from the database $V$ and $I$ band CMDs for these objects, using their coordinates and diameters to define a box extraction. Guided by DSS images of each system we selected representative field regions to extract stars and construct CMDs for comparison purposes. Ages and reddenings for the clusters were estimated by fitting the Padova isochrones. We adopted Bertelli et al.'s (1994) isochrones rather than the new isochrones by Girardi et al. (2000), since the previous set includes younger ages. The isochrone age grid suitable for $4,5,6.3,7.9,10,12,16,20,25,32,40,50,63,79$, $100,126,160,199,251,316,400,500,630,790$ and 1000 Myr. The isochrone age range and resolution allow 


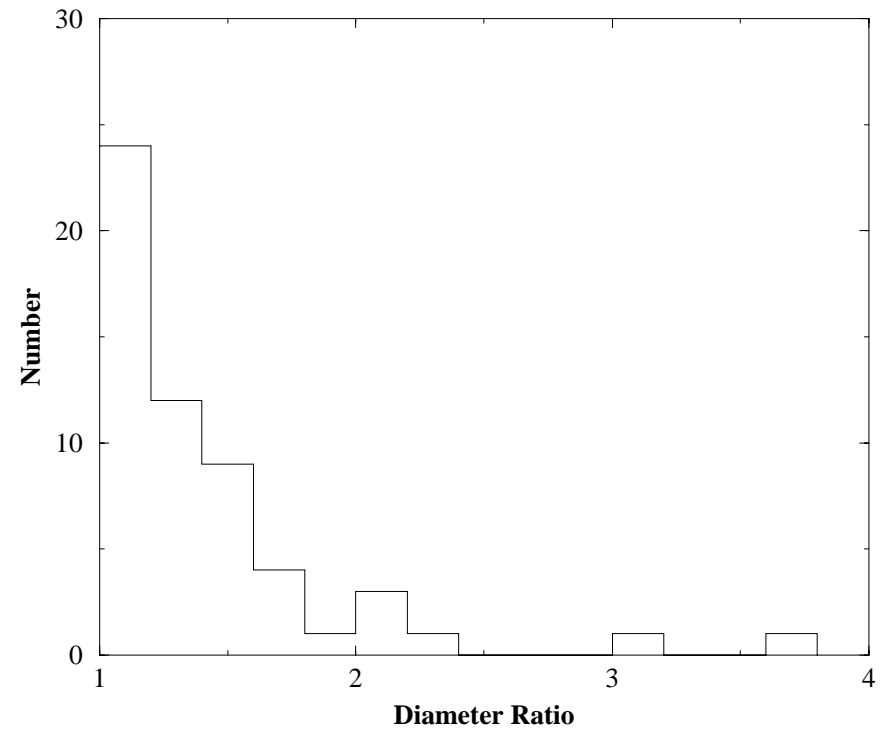

Fig. 10. The observed diameter ratio distribution for SMC cluster pairs



Fig. 11. Centre-to-centre angular separation distribution for SMC cluster pairs

one to estimate errors taking into account the stellar statistics in the cluster and field CMDs. We also assumed a SMC metallicity of $Z=0.004$, a foreground galactic reddening of $E(B-V)_{\mathrm{f}}=0.03$ in the SMC direction and $E(V-I) / E(B-V)=1.31$. Table 2 shows the ages, in Col. 2 and reddening values (foreground plus SMC internal) in Col. 3 determined in the present work via isochrone fitting. Ages and reddening values derived by Pietrzyński \& Udalski (1999b) are in Cols. 4 and 5 respectively, when available for comparisons. In our sample we could estimate CMD ages for 91 objects, 40 of them in common with Pietrzyński \& Udalski (1999b). We conclude that despite the reddening method and distance modulus differences between the two studies, there is good overall agreement for the age determinations. We also included ages for 21 objects embedded in HII regions, classified as NA and NC (see Col. 4 of Table 1, and also BS95). We assumed for them an age of 3 Myr.

\section{Discussion}

We overlap two age histograms in Fig. 8. The dotted line histogram shows objects from Table 2 with ages via CMDs. There are three peaks, at about 80, 220 and 450 Myr. The first two peaks appear to be present in Grebel et al.'s (2000) sample for 200 SMC clusters based on OGLE-II data, reported at $t \approx 100$ and 200 Myr respectively. They suggested them as enhanced star formation epochs. Considering Pietrzyński \& Udalski's (1999b) sample with CMD ages for $93 \mathrm{SMC}$ clusters, no peak is seen at $t=80-100 \mathrm{Myr}$, having their histogram a maximum at the youngest bin. In their histogram there occurs a local maximum at $t \approx 250-300 \mathrm{Myr}$, but the statistics is low. The third peak in the present study has no counterpart in both previous analyses, and it is probably an artifact from the low isochrone age step resolution in that range.

The solid line histogram in Fig. 8 shows additionally the embedded objects in HII regions for which we assumed an age of $3 \mathrm{Myr}$, and the objects from Table 2 with lower or upper limits by assuming them as the ages themselves. We can observe again a three peak distribution but the first peak is now shifted to the youngest bin similarly to Pietrzyński \& Udalski's (1999b) histogram. This suggests that only the $200 \mathrm{Myr}$ peak is relevant, being related to the SMC/LMC last encounter (Gardiner et al. 1994; Grebel et al.'s 2000). The maximum seen in the youngest bin is possibly related to the cluster formation/destruction rates. Since the present sample deals with pairs and multiplets a fast destruction rate might be caused by the internal dynamical evolution in each cluster complex, caused by merger and/or other effects.

Approximately $55 \%$ of cluster pairs and multiplets in Table 2 present similar ages between their members indicating that they are coeval. This suggests that most of the pairs and multiplets had a common origin, possibly from the same molecular complex. Note that about $60 \%$ of the pairs and multiplets embedded in OB associations (H-A, Hodge 1985), as indicated in Col. 9 of Table 1, have comparable ages between their members. This could be an explanation for the origin of cluster systems.

Considering triplet and multiplet members, we found that about $70 \%$ of them are younger than 100 Myr. These results suggest a possible binary cluster formation scenario: clusters can be born in multiplet systems and coalesce by mergers and tidal disruptions forming binary clusters in a timescale of $\approx 100 \mathrm{Myr}$. This time is in agreement with dynamical times required for an interacting pair to merge into a single cluster (ODB98, de Oliveira et al. 2000). 
The pairs with a bridge in the isophotal maps have comparable ages for their components (Table 2). As examples, the pairs NGC 241/NGC 242 and B39/BS30 in Fig. 2 show a bridge linking their members which could be interpreted as a sign of interaction (see the similarity with the N-body simulation model in Fig. 11 of ODB98). A typical timescale for the bridge phenomenon is $\approx 30 \mathrm{Myr}$, as deduced from a series of $\mathrm{N}$-body simulations related to bridge formation and evolution (ODB98).

Another interesting isophotal feature is related to the cluster triplet NGC 220/NGC 222/B23 which shows distortions for the small cluster in a direction almost perpendicular to the line connecting itself to the large components NGC 220 and NGC 222. This configuration and morphologies are compatible with a fast hyperbolic encounter with small impact parameter (e.g. Fig. 12 of ODB98).

Figure 9 shows the angular distribution of pairs and multiplets together with SMC HI column density isophotes from Mathewson \& Ford (1984). It can be seen that most of the objects are concentrated in the SMC main body, close to the higher concentration of HI, so it is not unexpected that in general they result young (Sect. 4). The objects appear to be distributed along an axis. Such distribution is present in the overall SMC cluster sample and there is growing evidence that it is related to a nearly edge-on disk containing the bulk of the young stellar population in the SMC (Bica et al. 1999; Westerlund 1990).

A nearly edge-on disk in a low internal reddening galaxy like the SMC would imply an increase of projection effects as compared to a simulation such as that carried out by Bhatia \& Hatzidimitriou (1988) for the nearly face-on LMC disk where the physical pairs would be about $50 \%$. Consequently the fraction of physical pairs in the SMC would be lower. The present approach including morphological evidence of interaction is an attempt to constrain this aspect. Indeed the fraction with isophotal distortions is only $25 \%$ (Sect. 3.1). Projection effects can be responsible for the age spread in some multiplets. For example the sextuplet (Table 2) has component ages 80 Myr (B72), 200 Myr (H86-143), 50 Myr (BS257), 3 Myr (SMC-N52A and SMC-N52B) and 400 Myr (H86-148). Possibly only the 3 or 4 younger components could be related to OB-Association $\mathrm{H}-\mathrm{A} 35$, the remaining objects would be captures or projection effects. This age spread is also present in IC 1611's quadruplet and in some triplets. On the other hand the quintuplet in the star forming complex NGC 395 has all its members with the same age (3 Myr) thus forming a physical system.

In Fig. 10 we show the distribution of the diameter ratio between members for all pairs in the sample. The diameter ratio is mostly in the range $1-2$, with a peak at 1 indicating that the majority of pair members have a comparable size. This effect was also observed in the LMC (Bhatia et al. 1991).
Figure 11 shows the distribution of the centre-to-centre angular separation between pair members. The separation range is $\approx 10-80 \operatorname{arcsec}(\approx 3-22 \mathrm{pc})$ with a pronounced peak at $\approx 45 \operatorname{arcsec}(13 \mathrm{pc})$. A similar peak was also observed by Bhatia et al. (1991) and de Oliveira (1996) who found a bimodal distribution for the LMC pairs with peaks at $\approx 5$ and 13 pc. The observed upper limit of the projected centre-to-centre linear separation $\approx 23 \mathrm{pc}$ (80 arcsec) is comparable to Bhatia \& Hatzidimitriou's (1988) separation criterion for pairs in the LMC (18.7 pc). The frequent separation value around $13 \mathrm{pc}$ may reflect a preferred survival distance for the systems, combined to projection effects.

\section{Conclusions}

We presented an isophotal atlas for 75 star cluster pairs and multiplets in the Small Magellanic Cloud, comprising 176 objects.

It was possible to derive ages from Colour-Magnitude Diagrams using the OGLE-II photometric survey for 91 objects. In addition we included in the analysis ages for 21 embedded objects in HII regions. The age distribution has a maximum in the youngest bin with a profile related to the cluster formation/destruction rates, in particular cluster multiple systems can have a fast destruction rate caused by their internal dynamical evolution. There is a second peak around $220 \mathrm{Myr}$ which is probably related to the SMC/LMC last encounter.

We find that $55 \%$ of the pairs and multiplets in the sample are in general coeval, indicating that captures are a rare phenomenon. Most of the cluster multiplets occur in OB stellar associations and/or HII region complexes which indicates a common origin and suggests that multiplets coalesce into pairs or single clusters in a short time scale $(\approx 100$ Myr $)$.

The majority of the cluster members have comparable sizes, with a diameter ratio ranging mostly between $1-2$. The projected separation distribution between the members of a pair has a pronounced peak at $\approx 13$ pc. These observational results are important constraints to theoretical models of star cluster pair encounters and could be related with the formation process and subsequent dynamical evolution of cluster systems.

The angular distribution of cluster pairs and multiplets shows that most of the objects are located in the SMC main body. The overall SMC cluster sample presents a similar distribution and there is evidence that it is related to a nearly edge-on disk in the SMC. Considering this, it is expected an increase of projection effects as compared to estimates for the LMC disk where physical pairs would be about $50 \%$ (Bhatia \& Hatzidimitriou 1988).

The atlas shows that around $25 \%$ of the isophote maps present relevant structures like bridges, common envelopes or detached distorted isophotes. N-body simulations have 
indicated that these structures arise from interactions between the members of the cluster systems. Indeed cluster pairs as NGC 241/NGC 242 and B39/BS30 show in their isophotal maps bridges linking their members and have comparable ages.

We conclude that multiplicity may have an important role in the early dynamical evolution of star clusters in general, and signatures of that may survive in the long term structure of large single clusters (de Oliveira et al. 2000).

Acknowledgements. We thank the referee Dr. B. Westerlund for interesting remarks. We acknowledge support from the Brazilian institutions CNPq, CAPES and FINEP.

\section{References}

Bertelli G., Bressan A., Chiosi C., 1994, A\&AS 106, 275

Bhatia R.K., Hatzidimitriou D., 1988, MNRAS 230, 215

Bhatia R.K., MacGillivray H.T., 1988, A\&A 203, L5

Bhatia R.K., Read M.A., Hatzidimitriou D., Tritton S., 1991, A\&AS 87, 335

Bica E., Schmitt H., 1995, ApJS 54, 33

Bica E., Clariá J.J., Dottori H., 1992, AJ 103, 1859

Bica E., Dutra C.M., 2000, AJ 119, 1214

Bica E., Schmitt H., Dutra C.M., Luz Oliveira H., 1999, AJ 117, 238

Brück M., 1976, Occas. Rep. R. Obs. Edinburgh 1, 1

Davies R.D., Elliot K.H., Meaburn S., 1976, MNRAS 81, 89

de Oliveira M.R., 1996, MSc Thesis, Universidade Federal do Rio Grande do Sul, Porto Alegre

de Oliveira M.R., Dottori H., Bica E., 1998, MNRAS 295, 921

de Oliveira M.R., Bica E., Dottori H., 2000, MNRAS 311, 589

Dieball A., Grebel E.K., 2000a, A\&A (in press)

Dieball A., Grebel E.K., Theis C., 2000b, A\&A (in press)

Gardiner L.T., Sawa T., Fujimoto M., 1994, MNRAS 266, 567

Girardi L., Bressan A., Bertelli G., Chiosi C., 2000, A\&AS 141, 371
Grebel E.K., Zaritsky D., Harris J., 2000, in News Views of the Magellanic Clouds. In: Proceedings of the IAU Symp. 190, You-Hua Chu, Suntzeef N.B., Hesser J.E., Bohlender D.A. (eds.), PASP Conf. Ser., p. 405

Hatzidimitriou D., Bhatia R.K., 1990, A\&A 230, 11

Henize K., 1956, ApJS 12, 163

Hodge P.W., 1960, ApJ 131, 351

Hodge P.W., 1985, PASP 97, 530

Hodge P.W., 1986, PASP 98, 1113

Kontizas E., Kontizas M., Michalitsianos A., 1993, A\&A 267, 59

Kron G.E., 1956, PASP 68, 125

Lauberts A., 1982, The ESO/Uppsala Survey of the ESO (B) Atlas, Munich: ESO

Leon S., Bergond G., Vallenari A., 1999, A\&A 344, 450

Lindsay E.M., 1958, MNRAS 118, 172

Mathewson D.S., Ford V.L., 1984, Structure and Evolution of the Magellanic Clouds. In: Proceedings of the IAU Symp. 108, van den Bergh S., de Boer K. (eds.). Kluwer, Dordrecht

Meyssonier N., Azzopardi M., 1993, A\&AS 102, 451

Pietrzyński G., Udalski A., 1999a, Acta Astron. 49, 165

Pietrzyński G., Udalski A., 1999b, Acta Astron. 49, 157

Pietrzyński G., Udalski A., Szymański M., et al., 1998, Acta Astron. 48, 175

Rodrigues I., Rodriguez A., Schmitt H., Dottori H., Bica E., 1994, Proceedings of the IIIrd ESO/CTIO Workshop on The Local Group: Comparative and Global Properties, Layden A., Smith R.C., Storm J. (eds.)

Sagar R., Richtler T., de Boer K.S., 1991, A\&A 249, L5

Shapley H., Lindsay E.M., 1963, Ir. Astron. J. 6, 74

Udalski A., 1998, Acta Astron. 48, 383

Udalski A., Kubiak M., Szymański M., 1997, Acta Astron. 47, 319

Udalski A., Szymański M., Kubiak M., et al., 1998, Acta Astron. 48, 147

Vallenari A., Bettoni D., Chiosi C., 1998, A\&A 331, 506

Westerlund B.E., 1990, A\&AR 2, 29 